The possibilities of reducing radiation dose and improve image quality in CT diagnostics using advanced image processing

Anne Catrine Trægde Martinsen

The Intervention Centre

and

The Department of Radiology and Nuclear Medicine

Oslo University Hospital

Faculty Division of Clinical Medicine

University of Oslo

Oslo, June 2011 
(C) Anne Catrine Trægde Martinsen, 2011

Series of dissertations submitted to the Faculty of Medicine, University of Oslo No. 1230

ISBN 978-82-8264-312-2

All rights reserved. No part of this publication may be reproduced or transmitted, in any form or by any means, without permission.

Cover: Inger Sandved Anfinsen.

Printed in Norway: AIT Oslo AS.

Produced in co-operation with Unipub.

The thesis is produced by Unipub merely in connection with the thesis defence. Kindly direct all inquiries regarding the thesis to the copyright holder or the unit which grants the doctorate. 


\section{List of contents}

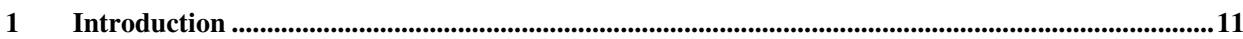

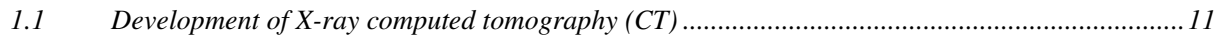

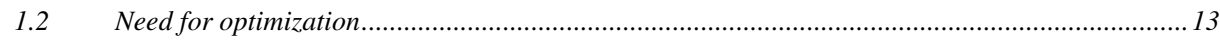

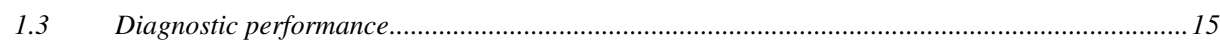

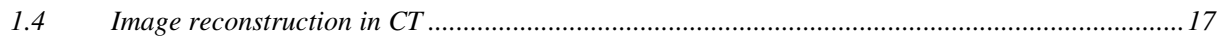

$2 \quad$ Aims of the study ...................................................................................................................................21

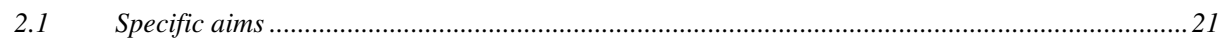

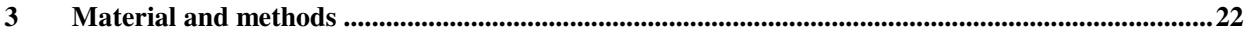

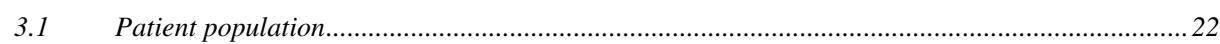

$3.2 \quad$ Methods

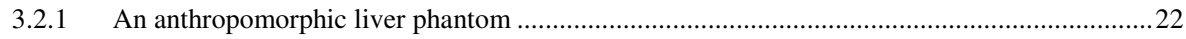

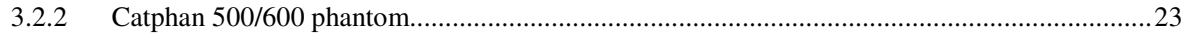

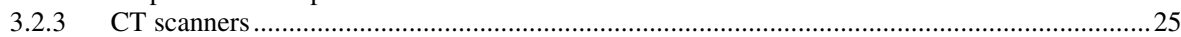

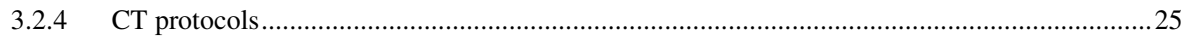

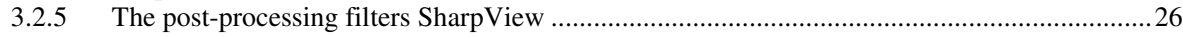

3.2.6 ASIR .

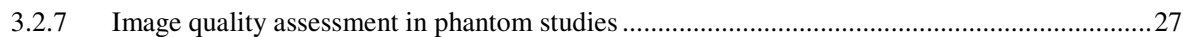

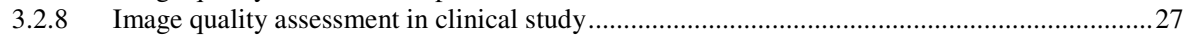

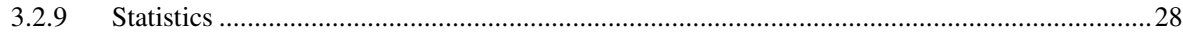

$4 \quad$ Summary of results, the individual papers.................................................................................................30

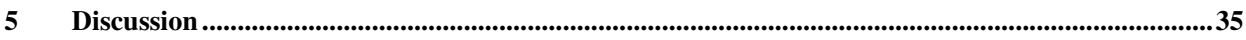

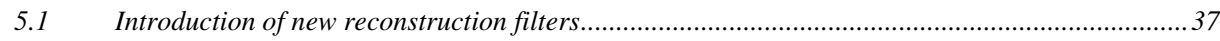

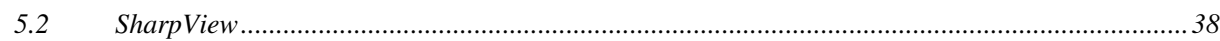

$5.3 \quad$ ASIR

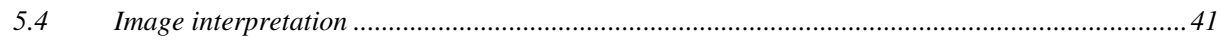

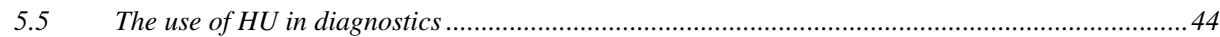

6 Conclusion ........................................................................................................................................4

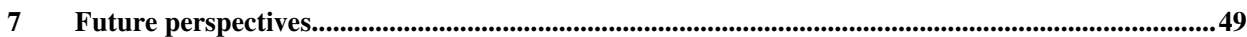

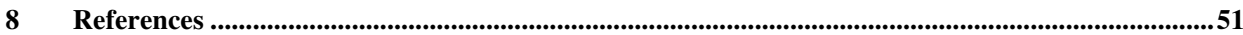

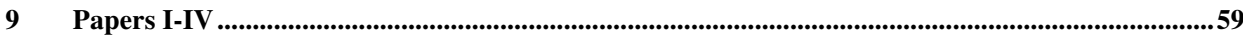




\section{Acknowledgement}

The present study was carried out during the years 2008 to 2011 at the Department of Radiology, Oslo University Hospital, Ullevål and at the Intervention Centre, Oslo University Hospital, Rikshospitalet.

I would like to express my sincere gratitude to my three supervisors Professor dr. med. Per Skaane, Professor dr. philos Dag Rune Olsen and Dr. med. Per Kristian Hol, with whom I have been delighted to work during this thesis. Their knowledge, support and guidance have been very valuable to my work. Professor dr. med. Per Skaane's experience and scientific knowledge have been important in my work with this thesis. Professor Dag Rune Olsen's knowledge, experience and work in the field of medical physics have been an inspiration to my work as a medical physicist in general, and in the work with this thesis in special. In 2010, Per Kristian Hol became co-supervisor. His experience, encouragement and guidance have been really valuable to me in the finishing part of the study.

I am very grateful to my co-authors: MSc Hilde Kjernlie Sæther, Dr ing Hilde Merethe Olerud, Professor dr. scient Eli Olaug Hole, Dr. Per Aage Wolff and MSc Erlend Peter Skaug Sande. It has been a pleasure to work with all of you. In special, I am very grateful to Hilde Sæther who has contributed essentially in three of the studies included in this thesis. Her knowledge, support and creativity have been most valuable in this work. Hilde Olerud's enthusiasm and skills in the field of CT and radiation protection have been an inspiration to my work with this thesis, and also as a medical physicist.

I am very grateful to the radiologists at the Radiological department at Oslo University Hospital, Ullevål; Dr. Catrine Elin Johansen, Dr. Hanne Grøstad, Dr. Knut Grønseth, Dr. 
med Johan Baptist Dormagen and Dr. Anders Drolsum for image interpretation and radiological advices to the study design. I also want to thank the participating $\mathrm{CT}$ radiographers at Ullevål; Mona Risdal and Kristin Livelten Heggen for their cooperation and support in the laboratory. Thank you for always being positive and for taking the time to help me in this study. It has been a great pleasure working with all of you.

My sincere thank to the whole staff at the Intervention Centre for support and inspiring working environment. A special thank go to all physicists in Section for Diagnostic Physics at the Intervention Centre. Their enthusiasm, knowledge and support have been most valuable to me. It is a pleasure working together with all of you! Special thanks to the readers in this study: Cand. scient Charlotte Kile Larsen, MSc Bjørn Helge Østerås, Dr. scient Øystein Bech Gadmar, MSc Jon Erik Holtedahl, MSc Hanne Blytt Andreassen, MSc Ingerid Skjei Knudtsen, MSc Anette Holvik and MSc Kristin Jensen.

Technical support from Anni Forsberg, Contextvision, has been valuable. I also would like to thank the statistician, Cathrine Brunborg for most helpful support.

I am grateful to the Head of the Clinic of Diagnostics and Intervention, Dr. med. Eli Marie Sager both for giving me the opportunity to establish a regional diagnostic physicist service and to encourage me to start the work on this thesis. Your encouragement and support have been most valuable to me. I would like to thank Turid Langli as former Head of the Radiological department, for giving me the possibility and support to start this work. I wish to express my gratitude to the Head of the Intervention Centre, Professor dr. med. Erik Fosse for the opportunity to finish this work. Your scientific knowledge, creativity and enthusiasm are an encouragement in my work. 
I want to thank my parents, Tove and Arne Kristian Pedersen, for always believing in me and supporting me.

Finally, a special thank to my dear husband and very best friend Øyvind Martinsen and our lovely children Kristin and Sondre, for all support and patience. You are my source of joy and love every day, and are showing me what is the most important in life. 


\section{Abbreviations}

ASIR Adaptive statistical iterative reconstruction

AUC Area under the ROC curves

CNR Contrast to noise ratio

CT Computed tomography

CTDI CT dose index

FBP Filtered back projection

HU Hounsfield Units

ICC Intraclass correlation coefficient

ICRP International committee on radiation protection

IR Iterative reconstruction

NRPA The Norwegian radiation protection authority

PACS Picture archiving and communication system

ROC Receiver operating characteristics

SNR Signal to noise ratio

SVCT SharpView CT post-processing filter

VGC Visual grading characteristic analysis 


\section{List of publications}

I. Martinsen ACT, Sæther HK, Olsen DR, Skaane P, Olerud HM: Reduction in Dose from CT Examinations of Liver Lesions with a New Postprocessing Filter: A ROC Phantom Study. Acta Radiol (2008) 49: 303 - 309.

II. Martinsen ACT, Sæther HK, Olsen DR, Wolff PA, Skaane P: Improved image quality of low dose thoracic CT examinations with new post-processing software. J Appl Clin Med Phys (2010) 11: 250-258.

III. Martinsen ACT, Sæther HK, Hol PK, Olsen DR, Skaane P: Itertative reconstruction reduces abdominal CT dose. Eur J Radiol 2011; DOI 10.1016/j.ejrad.2011.04.021 (published ahead of print)

IV. Sande EPS, Martinsen ACT, Hole EO, Olerud HM: Interphantom and interscanner variations for Hounsfield units - an establishment of reference values for HU in a commercial QA phantom. Phys Med Biol (2010) 55: 1-13. 


\section{Introduction}

\subsection{Development of X-ray computed tomography (CT)}

There has been a tremendous development in CT technology since its beginning in 1970's, with main focus increasing the scan speed and the image reconstruction ${ }^{1}$.

On $1^{\text {st }}$ October 1971 , the first CT scan of a patient was performed ${ }^{2}$. The first presentation of a CT scan of a human brain was lectured by the engineer Godfrey Hounsfield and Dr James Ambrose at the $32^{\text {nd }}$ Congress of the British Institute of Radiology in 1972. This new technique surprised the entire medical community. In fact, the first CT scanners were developed and manufactured by the record company, EMI Ltd, and not by any of the medical manufacturers. In 1979 Hounsfield, along with the physicist Alan McLeod Cormack, were awarded the Nobel Prize in physiology and medicine for their work ${ }^{1}$.

Electrical signals are generated as the x-rays transversing through the object scanned, are detected in the detector system. Electrical signals emitted from a set of projections make up a raw data set from which the CT images are reconstructed. The reconstruction algorithm, also known as "filtered back projection", is a mathematical procedure used for the convolution of the attenuation profiles and reconstruction of the CT image. Image texture, appearances and characteristics depend on the algorithm used.

The reconstruction matrix defines the pixel size. In the beginning the reconstruction matrix used was $80 \times 80$ and the corresponding voxel size was $3 \mathrm{~mm} \times 3 \mathrm{~mm} \times 13 \mathrm{~mm}$. In the first CT scanners, iterative reconstruction technique was used. The processing time of an image was 7 minutes with iterative reconstruction, and 30 seconds with 160 matrixes using 
filtered back projection ${ }^{2}$.To reduce the reconstruction time, filtered back projection (FBP) was used as a standard, and still FBP is the standard technique used for reconstruction in CT. In the early times, only brain scans were performed, due to long scan time and reconstruction time.

In the 1990s slip ring technology made spiral CT possible. Increase in tube power and higher computer capacity was also introduced. Spiral scanning improved 3D resolution and thus lesion detection, and also reduced scanning time ${ }^{3}$. In the 2000s multi detector row CT scanners were introduced, and today it is possible to scan 640 images pr rotation. 512 reconstruction matrixes are used as a standard, but some scanners also reconstruct in 768 and 1024 matrixes which give reduced voxel size. It is now possible to reconstruct $0.5 \mathrm{~mm}$ isotropic voxels. Whole body scans with isotropic volumetric data is accessed in less than 30 seconds.

Fast CT scans combined with advanced image reconstruction, post-processing and 3D reformatted images have revolutionized diagnostic X-ray imaging, as it provides vastly more diagnostic information than conventional X-ray imaging. Conventional X-ray procedures such as thoracic and abdominal examinations, coronary diagnostic imaging, and angiography, and x-ray fluoroscopy are nowadays substituted by CT examinations. New techniques like virtual colonography, organ perfusion and spectral imaging have recently been introduced. As a result of the rapid evolution of $\mathrm{CT}$, the number of CT examinations performed is rapidly increasing.

In the United States the number of performed CT examinations increased from 13 million scans in 1990 to 62 million scans in $2006^{4}$. According to the Norwegian Radiation Protection Authority (NRPA), the frequency of performed CT examinations increased from 
$6.5 \%$ of the total amount of performed radiological examinations in 1993 to $21.5 \%$ in 2008 .

Over the same period the number of conventional X-ray examinations was reduced from

$83.8 \%$ of the total amount of performed radiological examinations to $52.8 \%$ (figure 1$)^{5}$. In total 25.2 abdominal CT examinations per 1000 inhabitants and 24.3 thoracic CT examinations per 1000 inhabitants were performed annually in Norway in 2008.

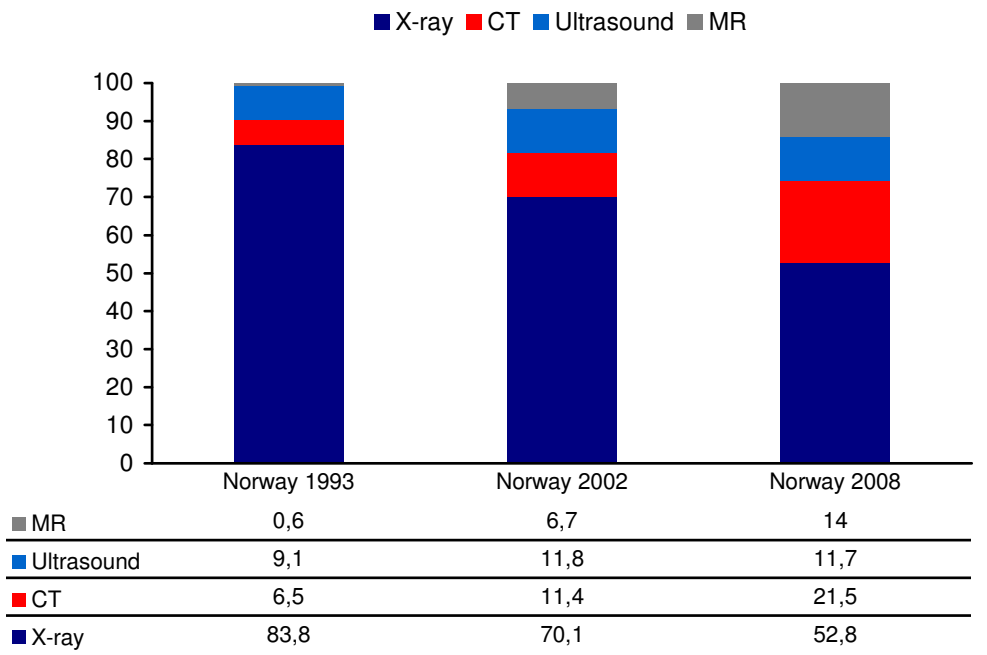

Figure 1: Percentage of CT, MR, X-ray and ultrasound examinations in Norway in 1993, 2002 and 20085.

\subsection{Need for optimization}

CT scans are associated with higher patient doses as compared to other radiological examinations. In European and US hospitals the CT examinations account for more than 50 $\%$ of the collective effective dose associated with medical exposure ${ }^{6,7}$. In $2002,65 \%$ of the total population radiation exposure in Norway was related to CT examinations increasing to $80 \%$ in $2008^{5}$. In Norway, CT examinations give rise to $59 \%$ of the total radiation dose associated with radiological examinations, but account for only $14 \%$ of the total X-ray 
examinations ${ }^{8}$. The European legislation demands that member states pay special attention to radiation protection in computed tomography ${ }^{9}$, and optimizing the CT examinations with respect to both radiation dose and image quality is mandatory in Norway. Optimizing the CT examinations is a balance between benefits and risks, and should favor the benefits. The ALARA (As Low As Reasonably Achievable) principle is considered a most useful concept in the prevention of stochastic effects such as cancer.

Radiation effects are divided into two groups; stochastic effects and deterministic effects. Stochastic effect in radiation is increased probability of cancer induction. Deterministic effects are in example cataract, hair loss and erythema. The International Committee on Radiation Protection (ICRP) states that for doses of $100 \mathrm{mSv}$ and higher, there is epidemiologic proven risk for radiation related cancer induction, and that there is no rational for assuming a low-dose threshold for cancer induction. In radiation protection management, it is therefore a general assumption that the risk for stochastic effects increases linearly with dose, without any threshold ${ }^{10}$. In the low dose range, normally used for radiological purposes, epidemiology does not directly prove increased risk of cancer induction. Therefore, the risk is estimated by extrapolation from evidence proved stochastic effects for higher dose levels, using the linear no-threshold model. The risk is assumed to reach $0.5 \%$ at an effective dose of $100 \mathrm{mSv}^{11}$. Sodickson et al. reported high rates of recurrent CT examinations: $33 \%$ of the patients included in their study underwent more than $5 \mathrm{CT}$ examination, 5\% underwent more than $22 \mathrm{CT}$ examinations and $1 \%$ underwent more than $38 \mathrm{CT}$ examinations. For $15 \%$ of these patients, the cumulative radiation dose exceeded $100 \mathrm{mSv}^{12}$. 
The organ doses from CT examinations including two or more scan series yields radiation doses above the threshold of $100 \mathrm{mSv}$. Between 1.5 and $2 \%$ of all cancers might be related to radiation from CT examinations ${ }^{6}$. In the $\mathrm{UK}$ about 700 cases of cancer annually is assumed attributed to diagnostic X-ray ${ }^{7}$. Berrington de Gonzales et al. estimated that nearly 29000 future cancers may be related to CT scans performed in the US in $2007^{13}$. In special, abdominal and thoracic CT examinations were highlighted as risk factors in their estimates.

The main goal in optimizing CT examinations is to reduce the radiation dose and at the same time maintain or even improve diagnostic accuracy. Adequate diagnostic image quality means that radiologists are able to distinguish between different states of disease and health, accurately detect and report relevant structures and features in the images or accurately classify different abnormalities in the images ${ }^{14}$. Accurate detection or exclusion of disease is crucial for appropriate treatment decision making.

Dose reduction techniques may decrease the image quality. Research on dose reduction strategies must therefore also include the diagnostic quality of the examination ${ }^{15}$. Different strategies for CT radiation dose and image quality optimization have been introduced: automatic current selection, bismuth shielding of breast tissue, thyroid gland and the lenses of the eyes, dose-reduction soft ware, use of different reconstruction filters and iterative reconstruction ${ }^{4,16-18}$.

\subsection{Diagnostic performance}

Modern CT scanners are becoming increasingly complex in both scanner- and detectordesign as well as reconstruction techniques. The manufacturers pursue different strategies in their development of the CT technology. There are differences in spectral energy, 
filtration of the x-ray tube and algorithms for cone beam reconstruction and postprocessing. This introduce inter-scanner and inter-manufacturer differences in CT images, for instance with respect to Hounsfield Units (HU). Each HU-value represents one shade of grey in the reconstructed image. Every pixel in a CT image is correlated to one specific $\mathrm{HU}$, which reflects tissue density because each pixel reflects the linear radiation attenuation coefficient of the corresponding volume element (voxel) ${ }^{19}$. HU is the relative difference in attenuation between tissue and water and the formula is:

$$
H U=1000\left(\mu_{\text {tissue }}-\mu_{\text {water }}\right) / \mu_{\text {water }}
$$

The various strategies in CT technology may influence the diagnostic performance differently. A diversity of imaging techniques, new reconstruction algorithms and postprocessing algorithms may result in images that do not look familiar to the radiologists with respect to grey-scale, noise suppression, linear structures, edge enhancement and variety in HU measurements.

In the 1980's, two studies concluded that absolute HU should not be used for clinical purposes $^{20-21}$. One of these studies, Zerhouni et al., concluded that relative rather than absolute HU should be used for diagnostic purposes ${ }^{21}$. Still, HU is often used for diagnostic purposes to differentiate between benign and malign adrenal masses and tissue and fluid characterization, to example in detection and staging of malignancy of adrenal masses, in quantitative analysis of lung cancer and in diagnostics of ovarian dermoid cysts ${ }^{22-31}$. In 2004, there was consensus that the uses of HU in gastrointestinal tumor density diagnostics can quantiate early tumor response evaluation of treatment. Correspondingly, Illias et al. claimed that "CT is the cornerstone imaging of adrenal tumors. Attenuation values of $<10$ 
HU on an unenhanced CT are practically diagnostic for adenomas ${ }^{29}$. For masses with attenuation $<10 \mathrm{HU}$ no further imaging is recommended, as they are considered to be benign ${ }^{29}$. Nieman states that the measurements of HU of the precontrast scan serie and contrast wash out in the venous phase are useful in adrenal diagnostics ${ }^{28}$.

Today, more scattering effects are induced with wider detector collimation. This might affect the HU, if not corrected for in the reconstruction algorithms. Still, the absolute HU is often used for diagnostic purposes.

\subsection{Image reconstruction in $\mathrm{CT}$}

The clinical desires for higher spatial resolution, increased low contrast detectability, greater volume coverage, and faster scan times and at the same time reduced radiation doses have forced the CT manufacturers to improve and develop new reconstruction algorithms and post-processing filters. Lately, a variety of iterative reconstruction algorithms has become available for clinical use that provides a better modeling of scanner geometry and physics ${ }^{32}$. In order to improve image quality without increasing the radiation doses, some third party manufacturers have developed stand-alone post-processing filters that are compatible with all commercially available CT scanners.

Filtered back projection $(\mathrm{FBP})$ reconstruction has been the standard reconstruction technique of CT images over the last 30 years and still is the gold standard for all modern

CT scanners ${ }^{33,34}$. FBP is a reconstruction model relying on the exact, mathematical relationship between measured attenuation in each projection and the corresponding pixel value in the image ${ }^{34}$. In FBP it is assumed that the focal spot is infinitely small and the dimension of the detector cells is ignored. 
Each of the projections from the scanner is noisy, and since exact data is assumed in FBP, noise is amplified in the image reconstruction process ${ }^{34}$. Data from each projection is assumed free of noise and equally valid due to FBP's mathematical simplifications, resulting in accumulation of noise in the reconstructed pixels ${ }^{35}$.

The iterative reconstruction (IR) model provides higher resolution and better robustness with respect to radiation scatter, motion artefacts, beam hardening artefacts and metal artefacts in the images compared to the FBP reconstruction model. Simplified, the iterative reconstruction is a mathematical trial and error procedure which gradually gives the correct answer $^{34}$. In IR the forward projection of the beam is synthesized and compared it to the actual measurement. The scanner geometry, shape of focal spot and detector cells, detector response and the geometry of the reconstruction image pixels are taken into account determined under image reconstruction ${ }^{36}$. There are two main types of iterative reconstructions: 1) The conventional algebraic iterative techniques solve a set of linear $\mathrm{x}$ ray beam attenuation equation. 2) The statistical iterative techniques use statistical modelling of noise information in the measured data in the reconstruction process ${ }^{33}$. Real iterative reconstruction gives higher spatial resolution, artefact and noise suppression compared to FBP in combination with post-processing filters. The drawback is increased reconstruction time. The statistical iterative techniques use statistical modelling of electronic and photon noise in the measured data in the reconstruction. No new information will appear in the data set after reconstruction except noise suppression. This method is less time consuming compared to the conventional algebraic technique. Today, the main benefit of iterative reconstruction techniques might be the possibility of improving the image quality such that significant radiation dose reduction is possible ${ }^{34}$. 
Post-processing filters, like "soft filters" may reduce the image noise, but they also reduce the spatial resolution and detectability of small structures and edges. At the same time edge enhancing filters improve the spatial resolution and detectability of dense, small structures and lesions in the images. On the other hand the low contrast detectability is reduced using this type of post-processing filters. To provide noise suppression, structure enhancement and detectability of small structures and edges different types of post-processing filters and reconstruction algorithms have been developed.

It is possible to reduce radiation doses using iterative reconstruction or post-processing filters $^{33,37-45}$. Information that is not present in the original data set will not be present in the post processed image either. Noise suppression may help the human eye to better reveal diagnostic information in the images. In many studies, only the signal to noise ratio (SNR) or contrast to noise ratio (CNR) is tested. As image post-processing or new algorithms are introduced, small structures, edges and lesions may potentially be suppressed as the noise is suppressed. Leander et al. showed that even though a new post-processing filter clearly reduced the image noise below that of a full dose images, visual grading analysis resulted in a superior result for the full dose images ${ }^{37}$.

Mayo et al. concludes that the complex relationship between radiation exposure, image noise and diagnostic accuracy should be investigated further to establish the minimum radiation dose that still provides an adequate diagnostic image quality ${ }^{17}$. Also the effect on HU should be evaluated.

One of the challenges in evaluating new imaging techniques is that the measure applied for "image quality" must reflect the radiologist's perception of good image quality. Receiver 
operating characteristics (ROC) analysis may be the most complete way of evaluating accuracy in a two group classification task and has proven to be a successful method to evaluate human reader performance under various imaging conditions ${ }^{15,46-49}$. 


\section{Aims of the study}

The main goal of this work was to assess the impact of reconstruction- and post-processing filters on diagnostic performance, and to evaluate the reliability of using absolute HU for diagnostic purposes.

\subsection{Specific aims}

Paper I: To evaluate the possibilities of reducing CT doses and at the same maintain or even improve the detection of small, simulated liver lesions at different dose levels, using a new post-processing mathematical filter, SharpView.

Paper II: To evaluate what impact a specific post-processing filter, SharpView, has on diagnostic performance on low dose CT thorax examinations.

Paper III: To assess the impact of an adaptive statistical iterative reconstruction (ASIR) on the detection of small simulated liver lesions at different dose levels.

Paper IV: To investigate and quantify interscanner variations in HU measurements and to assess possible differences in HU measurements between four supposedly identical Catphan phantoms. 


\section{Material and methods}

\subsection{Patient population}

In paper II a total of 13 colorectal cancer patients with suspected or known thoracic metastases were included in the study on the clinical impact of a new post-processing filter to improve image quality. The patients were included upon written informed consent. For five of the patients, the scan series did not cover all the chest and mediastinal area of interest and they were consequently excluded from the study. Four female and four male were included in the data analysis, ranging in age from 50 to 75 years; mean age was 67.6 years. The study was approved by the Regional Committees for Medical Research Ethics (REK) and the National Radiation Protection Authority (NRPA).

In paper I and III, a custom made anthropomorphic, upper abdomen phantom was used, and in paper IV four different Catphan 500/600 phantoms were used.

\subsection{Methods}

\subsubsection{An anthropomorphic liver phantom}

The custom made anthropomorphic, upper abdomen phantom (figure 2) is specially
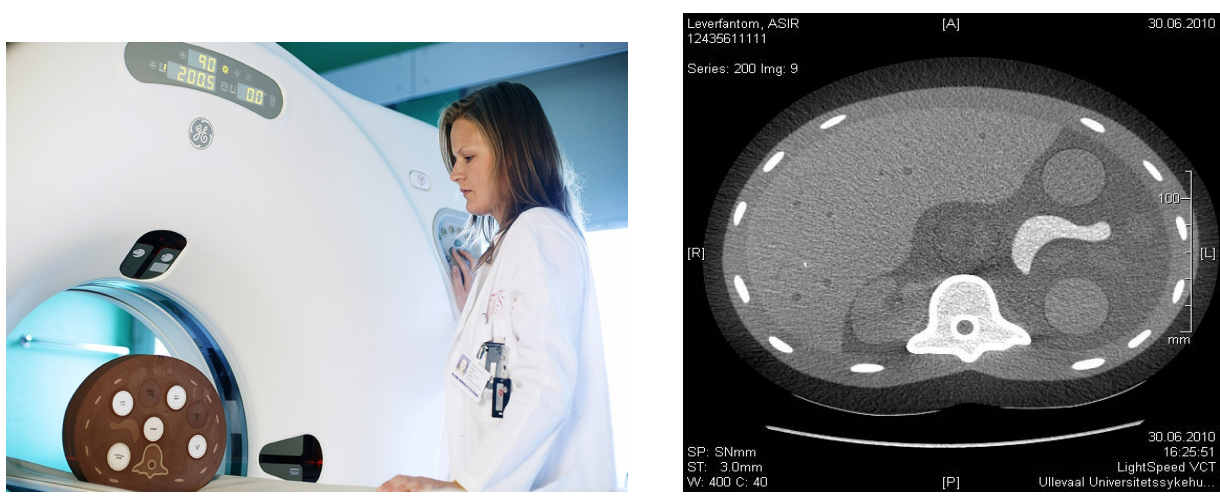

Figure 2:A custom made anthropomorphic, upper abdomen phantom specially designed for ROC studies. In the phantom are simulated liver lesions in the range 2-7 $\mathrm{mm}$. 
designed for ROC studies ${ }^{50}$. There are four liver tissue equivalent inserts in the liver part of the phantom. Each of these inserts is divided in 8 sectors. In each insert, there are holes with diameters in the range $2-7 \mathrm{~mm}$. In order to simulate liver lesions different solutions is filled in the holes. In total 32 predefined sectors are evaluated in the phantom; 16 sectors with holes and 16 sectors without. To avoid learning bias, these inserts were rotated in the cavity and also interchanged in the phantom.

\subsubsection{Catphan 500/600 phantom}

The Catphan 500/600 phantom from the Phantom Laboratory (The Phantom Laboratory, Salem NY, USA) is a CT quality assurance phantom suitable to test low contrast detectability, spatial resolution, noise, slice thickness and homogeneity. The phantom is divided in different test modules (figure 3). In this thesis, the sensitometry modules CTP 401 (Catphan 500) and CTP 404 (Catphan 600) were used. These modules are suitable for HU measurements. CTP 404 has inserts made from teflon ${ }^{\circledR}$, delrin ${ }^{\circledR}$, acrylic, polystyrene and low density polyethylene (LDPE), polymethylpentene (PMP) and air. In CTP401 there are only teflon, acrylic and low density polyethylene (LDPE) and air targets ${ }^{51}$. inserts are in the range $-1000 \mathrm{HU}$ (air) to $+990 \mathrm{HU}$ (teflon). 


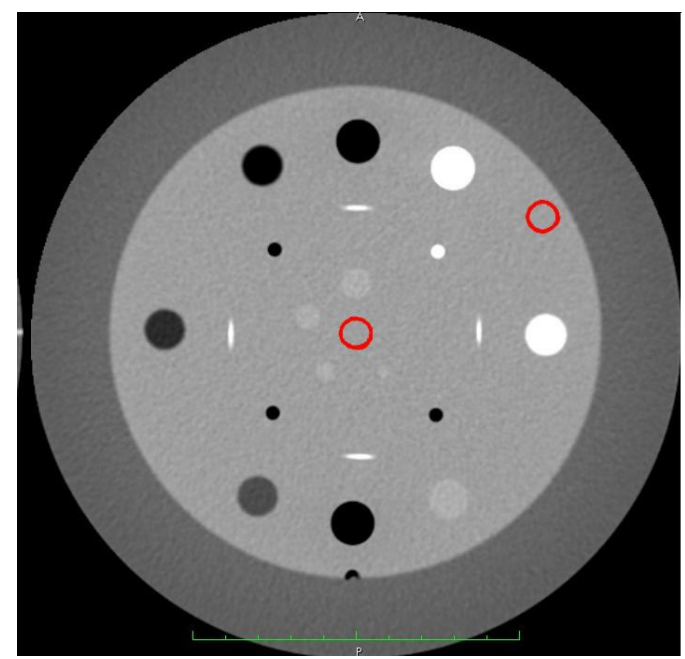

Figure 3: Catphan 600, module CTP 401 used for sensitometry measurements.

Different Catphan 500/600 phantom images were obtained on one CT scanner, to assess potential inter phantom variations in HU. "Fast calibration" and "tube warm up" were performed before scanning, to ensure stable energy and proper detector calibration during the test scans. Module CTP 401/404 was imaged at different scanners. The mean HU together with corresponding standard deviation were measured by placing a $60 \mathrm{~mm}^{2}$ region of interest (ROI) within the inserts in the CT images. For all the inserts, a mean value for all the phantoms was estimated.

The phantom that best represented the mean HU reading of all the phantoms was chosen for HU measurements of different CT scanners. The abdominal reconstruction algorithm of each scanner was used in this study, since diagnostic discrimination between malignant and benign tissue using HU, mostly are performed in the abdominal area. Test was performed at $80 \mathrm{kV}, 120 \mathrm{kV}$ and $140 \mathrm{kV}$. 


\subsubsection{CT scanners}

CT scanners from all manufacturers on the Norwegian market were included in this study; a four-slice GE Lightspeed QXi (GE Medical Systems, Milwaukee, Wisc., USA) in paper I, a 64-slice Philips Brilliance scanner (Philips Medical Systems, Best, The Netherlands) in paper II and a 64-slice GE Lightspeed VCT (GE Healthcare, Milwaukee, Wisc., USA) in paper III. In paper IV 64-slice CT scanners from all manufacturers on the Norwegian market were tested; Philips Brilliance 64, Toshiba Aquillion 64 (Toshiba Medical systems, Tokyo, Japan), Siemens Somatom Sensation 64 (Siemens Medical Solutions, Erlangen, Germany) and GE Lightspeed VCT. Also 16-slice CT scanners from GE and Philips were tested.

\subsubsection{CT protocols}

In both paper I and III the standard abdominal CT protocol from Department of Radiology, Oslo University Hospital Ullevål, was used.

In the clinical study both the full dose (reference dose $200 \mathrm{mAs}$ ) and the low dose (30 $\mathrm{mAs}$ ) thoracic CT protocols were used. These protocols are the standard protocols used for standard and low dose thoracic CT examinations at the Oslo University Hospital, Ullevål. Automatic exposure control was used for the full dose scan series for all patients. Both automatic current selection (ACS) and modulation in the Z-direction (Z-DOM) was used for all patients. The functionality of ACS and Z-DOM is described in a previous study ${ }^{52}$. On the Philips Brilliance 64 CT scanner it is not possible to scan with 3D modulation, so angular current adjustment was not used in our study. 
All CT protocols used, had been optimized with respect to adequate diagnostic image quality and acceptable dose level, by radiographer, radiologist and physicist beforehand. The $\mathrm{CTDI}_{\mathrm{vol}}$ were $11.9 \mathrm{mGy}$ and $8 \mathrm{mGy}$ for the standard abdominal and standard thoracic CT examinations used at the hospital, respectively. In comparison, the national reference levels for abdominal and thoracic CT examinations are $25 \mathrm{mGy}$ and $20 \mathrm{mGy}$, respectively ${ }^{53}$. The recommended CTDIvol in the EU guidelines for abdomen and chest are 15 and 10 mGy respectively, which is higher than the doses used in this study ${ }^{9}$.

\subsubsection{The post-processing filters SharpView}

SharpView CT (ContextVision AB, Linkoping, Sweden) is a stand-alone product and is compatible to all CT scanners regardless of manufacturer or mode ${ }^{54,55}$. SharpView CT consists of an adaptive filter bank controlled by image content. The adaptive filter is applied as a post-processing step after standard filtered back projection reconstruction. Structures, lines and edges are recognized by a pixel-to-pixel examination, and the system decides whether each pixel is a part of a linear structure based whether each neighbor's pixel is part of the same structure. The enhancement processing is performed in different intensity value ranges, corresponding to tissue type-specific Hounsfield units $(\mathrm{HU})^{37,55}$.

\subsubsection{ASIR}

The ASIR technique blends FBP techniques and statistical iterative reconstruction to obtain different levels of noise reduction in the images. The image reconstruction is performed from the raw data with both FBP and iterative techniques. A weighted summation of each data is performed for final image reconstruction. 50\% ASIR means a blending of $50 \%$ FBP reconstruction and 50\% ASIR data with a corresponding predicted 50\% noise reduction 
level ${ }^{33}$. In paper III, 50\% ASIR was used for all dose levels. The full dose FBP images were used as gold standard.

\subsubsection{Image quality assessment in phantom studies}

The readers independently assessed a set of images obtained at different dose levels to be evaluated on a 5-point scale; Score 1: Definitely negative, Score 2: Probably negative, Score 3: Possibly positive, Score 4: Probably positive, Score 5: Definitely positive.

All images were presented in a randomized manner with respect to dose and reconstruction techniques to the observers. All readers were trained on the phantom images, inserts, lesions assessment and grading criterion before image interpretation started, such that the evaluation system was well known.

\subsubsection{Image quality assessment in clinical study}

The impact on diagnostic performance of introducing the post-processing filter, SharpView was evaluated in a clinical study.

Two thoracic scan series were performed on colorectal cancer patients: one standard thoracic CT scan and one low dose thoracic CT scan. The low dose images were post processed with SharpView. Experienced CT radiologists assessed one full dose series with standard reconstruction, one low dose series with standard reconstruction and one low dose series with SharpView post-processing.

All images were presented in randomized and blinded manner. The image quality was evaluated using visual grading characteristics analysis (VGC). VGC analysis were 
performed on a five-point scale with respect to eight image quality criteria chosen from general chest and high resolution CT (HRCT) of the European guidelines on quality criteria

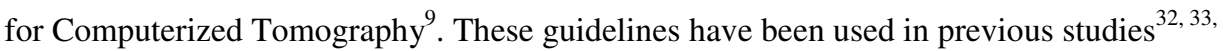
37, and 56 . The readers evaluated each criterion according to their individual perceptions by using the five-point scale: Score $1=$ not visible, score $2=$ poor -hardly visible, score $3=$ visible, score 4= clearly visible, score 5=visually sharp.

\subsubsection{Statistics}

Receiver operating characteristics (ROC) methodology was pursued to evaluate the diagnostic performance of ASIR and Sharpview compared to the standard reconstruction normally used. The ROC curves were derived by using Analyse-IT (Version 1.72, Jul 22 2004, Analyse-it software Ltd, United Kingdom).

A paired sampled t-test with a $95 \%$ confidence interval was used to compare diagnostic performance of standard images compared to post processed image.

Visual grading analysis (VGA) was used to evaluate the image quality, and an intra-class correlation (ICC) test with $95 \%$ confidence interval was used to evaluate inter observer differences. Definitions on levels of agreement were as follows: ICC $=1$ corresponded to complete agreement between the observers, and $\mathrm{ICC}=0$ corresponded to no agreement at all between the observers.

Intra-observer differences were not evaluated in this thesis, because all readers assessed the images only once to avoid learning bias. 


\section{Summary of results, the individual papers}

\section{Paper I}

Reduction in Dose from CT Examinations of Liver Lesions with a New Postprocessing Filter: A ROC Phantom Study. Martinsen ACT, Sather HK, Olsen DR, Skaane P, Olerud HM. Acta Radiol (2008) 49: 303 - 309

The aim of this study was to evaluate the possibilities of reducing the radiation doses from liver CT examinations and at the same time maintain the diagnostic performance using a new post-processing filter named SharpView (Context Vision AB, Sweden).

The standard abdominal CT protocol from the Department of Radiology, Oslo University Hospital Ullevål, was used. Images at five different dose levels were obtained. At all dose levels the images were reconstructed both with standard FBP reconstruction and filter normally used for the abdominal CT protocol, as well as for the new filter. Detection of simulated hypo-dense liver lesions, in the range of 2-7 mm, in an anthropomorphic upper abdomen phantom was evaluated. Six readers independently assessed a set of 10 images obtained at five different dose levels on a 5-point scale. ROC methodology was pursued to evaluate the diagnostic performance of Sharpview compared to the standard reconstruction normally used.

Improved diagnostic performance of abdominal CT examinations followed by SharpView post-processing was seen at a dose reduction of more than $30 \%$. The overall diagnostic performance was higher for SharpView post-processed images compared to the standard reconstruction. SharpView improved the diagnostic performance at all dose levels compared to the standard reconstructed images. Also, the inter-observer differences were 
reduced using SharpView post-processing compared to the standard reconstruction, at all dose levels.

It was concluded that there is a significant potential for reducing doses of CT liver examinations up to $30 \%$ whilst maintaining or even improving the diagnostic performance in the detection of 2-7 mm liver lesions by using SharpView post- processing.

\section{Paper II}

Improved image quality of low dose thoracic CT examinations with new post-processing software. Martinsen ACT, Sather HK, Olsen DR, Wolff PA, Skaane P. J Appl Clin Med Phys (2010) 11: 250-258.

The aim of this study was to evaluate the clinical benefit of using the SharpView postprocessing filter for thoracic low dose CT examinations.

Eight colorectal cancer patients were included in the study; four male and four female, ranging in age from 50 to 75 years; mean age was 67.6 years. Thoracic CT was performed as part of standard follow-up regime for colorectal cancer patients. Each patient underwent one standard, full-dose thoracic CT examination and one low-dose thoracic CT examination. All scans were performed on a Philips Brilliance 64-slice CT scanner.

Three radiologists evaluated 24 scan series with respect to image quality according to quality criteria from the European guidelines for chest CT. Each reading session consisted of one scan series for each patient, randomized with respect to dose levels, such that a reading session consisted of full dose series for some patients, low dose series for some patients and low dose SharpView enhanced series for some patients. Image reading 
included three reading sessions over a period of time long enough to avoid recognition of the pathology appearing on the images.

Image quality of the full-dose series scored significantly higher as compared to both the low-dose series and SharpView post-processed low-dose series (3.8 versus 3.0 and 3.3 respectively, $p<0.05)$. The average score for all details rated for all three readers were higher for the low-dose images with SharpView post-processing compared to the low-dose series with standard reconstruction (3.3 versus 3.0, respectively, $p<0.05)$. Overall mean score is the average score for all details rated for all three readers.

This paper concluded that there is a potential of improving the diagnostic performance of low-dose CT thoracic examinations using SharpView post-processing filter optimized with both respect to anatomical region of interest and dose levels.

\section{Paper III}

Iterative reconstruction reduces abdominal CT dose. Martinsen ACT, Saether HK, Hol PK, Olsen DR, Skaane P. Eur J Radiol 2011; DOI 10.1016/j.ejrad.2011.04.021 (published ahead of print)

The aim of this study was to evaluate the possibilities of reducing the radiation doses of liver CT examinations and at the same time maintaining the diagnostic performance using an adaptive statistical iterative reconstruction technique (ASIR).

The CT scans were performed with the standard abdominal CT protocol from the Department of Radiology, Oslo University Hospital Ullevål on a GE Lightspeed VCT 
scanner. Four readers independently assessed a set of 21 images obtained at six different dose levels on a 5-point scale. A blending of 50\% ASIR and 50\% filtered back projection (FBP) reconstruction was used at all dose levels. ROC methodology was pursued to evaluate the diagnostic performance of ASIR compared to the standard reconstruction technique normally used.

No difference in diagnostic performance was detected using ASIR compared to the full dose (250 mAs) FBP reconstructed images, except at $100 \mathrm{mAs}$. No significant difference in diagnostic performance between different dose levels for FBP reconstruction was detected, except for the two lowest dose levels (120 and $100 \mathrm{mAs})$. The diagnostic performance was better for different blending of $\operatorname{ASIR}(50,60,80$ and $90 \%)$ at $100 \mathrm{mAs}$ compared to the standard FBP reconstructed image, except for 70\% ASIR . No improvement in diagnostic performance at the highest dose levels using 50\% ASIR was detected.

In conclusion a blending of 50\% ASIR and FBP may improve image quality of CT examinations of the liver, and thus yield a potential for reducing radiation dose.

\section{Paper IV}

Interphantom and interscanner variations for Hounsfield units—an establishment of reference values for HU in a commercial QA phantom. Sande EPS, Martinsen ACT, Hole EO, Olerud HM. Phys Med Biol (2010) 55:1-13.

The aim of this study was to investigate inter-phantom and inter-scanner variations in Hounsfield units (HU) measurements, and also to establish a set of energy-specific HU reference values for the density module of the Catphan 500/600 phantom. 
Four different Catphan CT phantoms were scanned on one GE Lightspeed VCT scanner to evaluate possible inter-phantom variations in HU. $8 \mathrm{CT}$ scanners from 4 different manufacturers were evaluated.

The interphantom variation were small; in the range 2-5 HU. The inter-scanner variations were large; in the range 7-56 HU depending on spectral energy and density of the test materials in the Catphan phantom. Substantial deviation were found in materials with HU close to that of water, like Acrylic, polystyrene and LDPE. One manufacturer deviated substantially from the others with respect to the HU measurements.

We conclude that the use of scanner independent absolute $\mathrm{HU}$ in tissue and fluid characterization should be used with caution since substantial inter-scanner variations in HU was found in this study. Only relative HU should be used in tissue and fluid characterization, for instance the difference in HU between organs or different fluids. 


\section{Discussion}

The number of performed CT examinations have grown rapidly over the last decades ${ }^{4,5}$ as a consequence of new technological advances. There is no doubt that CT provides great medical benefits. Still, one drawback is the relatively high radiation doses from CT examinations. In some years, effects of ionizing radiation to the humans have been discussed all over the world in media, among medical personnel and among patients. Potential risks of radiation-induced cancers have become a hot topic with special attention on CT examinations. It is important that the radiologists are aware of these effects, and are weighing the diagnostic benefits against the risk. Based on these facts, the European Society of Radiology stated that "Radiation protection has become a top priority" in $2011^{11}$. Earlier, it has been claimed that "Radiologists have not focused their efforts sufficiently on radiation dose reduction" ${ }^{15}$. However, over the last decade there has been an increased focus on radiation protection. According to Coakley et al., we are now in a new wave of radiation consciousness, since the publications in the last years are indicating that CT doses cause an increase in radiation induced cancer ${ }^{57}$. There has been radiation consciousness in Europe for many years, but this consciousness is now seen in the North America as well ${ }^{57}$. The international effort, "Image gently" is introduced, aiming to reduce radiation doses ${ }^{15}$. In our hospital, $\mathrm{CT}$ radiation doses are routinely reported for each scan series and each examination and stored in the PACS. Dose reports on abdominal and thoracic CT radiation doses are reported to the Norwegian Radiation Protection Authority.

Both justification and optimization of CT examinations is warranted and is also mandatory in Norway according to national legislation. The main goal of optimizing CT examinations is to reduce the radiation dose and at the same time maintain or even improve diagnostic 
accuracy according to the ALARA principle. Different approaches have been introduced to achieve lower radiation dose and maintain adequate diagnostic image quality by the CT vendors. Automatic tube current modulation, low $\mathrm{kVp}$ imaging techniques, more efficient detector systems, more efficient collimators and improved image reconstruction filters including iterative reconstruction techniques are some of the features introduced over the last decade.

Reduction in tube current is one way to achieve lower radiation dose, since the radiation dose and tube current is linearly related. Normally, the image noise is increasing and low contrast detectability are decreasing as the tube current is reduced, due to decreased number of photons. Therefore, new reconstruction methods are necessary to achieve dose reduction without compromising image quality. In special, iterative reconstruction techniques have recently been reported in the literature as a powerful tool in image optimizing in CT. The drawback of iterative reconstruction technique is prolonged reconstruction time $\mathrm{e}^{32,34-35,39,41-45}$, which is also the reason why this has not been introduced earlier.

The results of this thesis demonstrate the potential for improving the diagnostic performance of low-dose $\mathrm{CT}$ using new adaptive iterative reconstruction or adaptive postprocessing filters, like ASIR and SharpView (paper I-III). Also other filters, like ANR-3D and prior image constrained compressed sensing (PICCS) potentially allow dose reduction without compromising image quality ${ }^{58,59}$. It is, however, important to notice that such filters may affect image texture and measured HU. Substantial inter-scanner variations in HU were detected in this study (paper IV). Due to this fact, relative rather than absolute HU values are recommended in clinical diagnostics. 
The image quality of CT examinations of obese patients might be insufficient, due to decreased number of photons reaching the detector. Therefore, these new filters may also be useful tools in imaging of obese patients as image noise is suppressed ${ }^{41}$.

\subsection{Introduction of new reconstruction filters}

Both SharpView post-processed images and the ASIR reconstructed images appeared different to the radiologists as compared with the conventional FBP techniques. Some of the readers in this study commented that the filtered images looked unfamiliar or "strange". Substantial changes in image noise texture might be uncomfortable to the radiologists ${ }^{32}$. This may discourage the implementation of new techniques, because the altered appearance may make radiologists uncertain with regard to the diagnostic performance.

We observed that the effect of both SharpView and ASIR was minor in the full dose images. In the full dose images, the noise level is already low and noise suppression might not lead to improved image quality. Suppression of the noise in the full dose image may further give too strong effect on the image texture and image appearance due to oversmoothing. Less filtering might give a better diagnostic performance at the higher dose levels. This is also in accordance with results from Leipsic et $\mathrm{al}^{42}$. They discovered a degradation of image quality due to different noise texture and smoothed borders for $100 \%$ ASIR compared to lower blending of ASIR and standard FBP reconstruction ${ }^{42}$. Correspondingly, Leander et al concluded that even though the noise level in SharpView post-processed low dose images was lower than the noise level in the full dose series, the diagnostic performance had a higher score for the full dose series ${ }^{37}$. This may indicate that a possible dose reduction cannot be predicted from improved signal-to-noise ratio alone, 
which has been claimed by other authors ${ }^{39}$. Our results support the need for performance studies, before new image reconstruction techniques are introduced into clinical practice as already suggested by Leipsic et $\mathrm{al}^{42}$ and Leander et $\mathrm{al}^{37}$.

In general, as shown in this thesis, the diagnostic performance of the filtered images had an overall higher score compared to the standard images at the same dose level. In paper I, it was demonstrated that a dose reduction up to $30 \%$ was possible when SharpView postprocessing was performed on liver images. In paper III, it was demonstrated that ASIR improved image quality for low dose images significantly. These results indicate that even though the images appeared "different" and unfamiliar to the radiologists, the detection of small low contrast structures and details were improved using new filters.

\subsection{SharpView}

This thesis confirms that there is a great potential for achieving a reduction in dose using SharpView post-processing filter. At the time our study started, no other studies regarding the Sharp View post-processing filter was reported. In 2009, Kröpil et al. published evaluations of the SharpView filter and in 2010 Leander et al. published their evaluations of the Sharp View post-processing. Both these studies were supporting our results.

Leander et al concluded that the SharpView post-processing filter improved image quality and reduced the noise level of abdominal CT examinations ${ }^{37}$. Still, the VGC analysis of the full dose series was superior to that of the low dose series. This is in accordance with the results in paper II. The result of VGC analysis of thoracic full dose series was superior to that of the low dose series. VGC analysis of the low dose series post-processed with SharpView was superior to that of the standard low dose series. 
The results of paper I indicate potential dose reductions for liver examinations of $30 \%$ using SharpView post-processing filter. Kröpil et al concluded that a dose reduction of up to $50 \%$ would be possible for upper abdominal CT examinations using SharpView postprocessing $^{40}$. Similar to those methods used in paper I, Kröpil et al used an anthropomorphic upper abdomen phantom to assess image quality following SharpView post-processing. Different structures in their images were rated on a 5-point scale in order to describe the diagnostic quality of the images. The full dose levels Kröpil et al refer to $\left(\mathrm{CTDI}_{\mathrm{vol}} \geq 20 \mathrm{mGy}\right)$ are nearly twice the full dose level used in paper I $\left(\mathrm{CTDI}_{\mathrm{vol}}=11.9\right.$ $\mathrm{mGy})^{40}$. The thoracic CT protocol in our hospital was optimized with respect to image quality and dose prior to our studies. This may explain why a larger dose reduction is demonstrated in other studies published as compared to the work presented in this thesis.

Third party post-processing filters, like SharpView, are working on already reconstructed images following CT image acquisition and prior to archiving in the PACS system. Even though the image texture may change using these filters, there is no new information added to the data set of the post-processed images compared to the FBP data. Noise suppression may improve the low contrast detectability in the images, but may also hide small pathological structures, decrease lesion conspicuity and lesion to background contrast. The frequency of spatial resolution is the same as the frequency of noise in the Fourier space, and noise suppression may mistakenly suppress small structures in the images. This is supported by Kalra et al. who concluded that noise-reduction filters decreased lesion contrast and conspicuity ${ }^{18}$. Also Hara et al. reported reduced spatial resolution with adaptive statistical iterative reconstruction compared to $\mathrm{FBP}^{45}$. 
Thus, tests like ROC- or VGC-analysis should be performed before new techniques are implemented clinically to evaluate potential improvements compared to conventional and established techniques. Also, it is important that the radiologists are aware of the differences between conventional and new techniques, like differences in grey-scaling, differences in detecting small lesions and structures, differences in image texture, and differences in $\mathrm{HU}$ for certain tissues. The introduction of new techniques may therefore also demand the need for new image interpretation regimes.

SharpView is a stand-alone product which according to the vendor, may be used on CT scanners from all CT vendors. Both Leander's and Kröpil's studies were performed on Siemens CT scanners, while the study in this thesis was performed on a GE four-slice scanner (paper I ) and a Philips 64-slice scanner (paper II). The results from our studies are in accordance with Leander's and Kröpil's findings and confirm that SharpView can improve the image quality regardless of CT manufacturer or model.

\subsection{ASIR}

This thesis demonstrates that ASIR may reduce the dose without compromising the image quality. Previous studies have also come to similar conclusions $32,35,39,41-45$. Paper III concluded that a blending of 50\% ASIR and FBP may improve image quality of CT examinations of the liver, and thus provide a potential for reducing radiation dose.

Other studies published recently support the conclusion in paper $\mathrm{III}^{32,35}$. Some studies have tested contrast-to-noise-ratio, signal-to noise or noise power ${ }^{39}$. In this thesis, the perception of low contrast objects was measured. A higher level of image noise would reduce diagnostic performance significantly. Singh et al. tested the diagnostic performance at 
approximately the same dose levels as we did, and their conclusion was that ASIR lowers noise and improves diagnostic confidence for subtle abdominal lesions ${ }^{35}$.

Silva et al. stated that ASIR allows more aggressive dose reduction with ultra-low dose techniques. The mAs-settings for CT colonography were reduced from $50 \mathrm{mAs}$ to $25 \mathrm{mAs}$ using ASIR. Silva et al. concluded that the use of ASIR has important implications both for screening $\mathrm{CT}$ and routine $\mathrm{CT}$ imaging. Leipsic et al. suggested that ASIR gives a possible dose reduction of $44 \%$ compared to FBP while preserving adequate image quality in coronary CT angiography ${ }^{43}$. As expected, the potential for dose reduction using ASIR is largest for patients with low $\mathrm{BMI}^{45}$. For patients wit $\mathrm{BMI}<20$ the potential dose reduction was $64 \%$ compared to potential dose reduction of $35 \%$ for patients with BMI> $25^{45}$. Still, CT with ASIR performed on obese patients may reduce the image noise and improve image quality as reported in the literature ${ }^{43}$.

Sagara et al. concluded that the image quality in low-dose abdominal CT scans with $40 \%$ ASIR is nearly comparable to that of full-dose techniques with FBP reconstruction alone. In their hospital, ASIR is fully integrated into clinical practice. All abdominal CT scans in their hospital are performed as low-dose abdominal CT scans reconstructed with 40-50\% $\mathrm{ASIR}^{35}$

\subsection{Image interpretation}

Quality assurance phantoms are commercially available; these phantoms do not necessarily simulate human tissue and may not be suitable for measure diagnostic performance. To our knowledge, none of these phantoms are specially designed for measuring the diagnostic performance with ROC analyses. In paper I and III a custom made, anthropomorphic upper 
abdomen CT phantom was used. In this phantom 32 test sectors are evaluated to yield the ROC curves. All readers were assessing these test sectors giving scores on a five-point scale. To improve the significance level of the results, a wider scale or more images from each dose level could have been used. The readers commented that test sectors were sometimes difficult to detect, especially at the lowest dose levels. This may introduce uncertainty in the results. One physicist was attending all the reading sessions, to note the results. Then the readers could solely focus on the images on the screen and concentrate only on the test sectors.

Simulated hypo-dense liver lesions were tested. A liquid glycerol solution was used in the insert (paper I). Most liver lesions are hypo-dense in non-iodine enhanced scan series. The HU values measured in these lesions are lower than HU measured in the liver tissue, corresponding to the liquid glycerol solution used in liver inserts in paper I. Liver cysts normally have the same density as water $(\mathrm{HU}=0)$. In paper III, the density of the liver inserts corresponded to that of liver cysts, since water was used in the inserts to avoid problems with air bubbles and precipitation. To fully assess the impact of ASIR and SharpView on diagnostic performance, also hyper-dense inserts should be tested in future studies.

All images in paper I-III were randomized with respect to dose level and blinded, such that the readers did not know if they were assessing standard images or post-processed images. Still, both ASIR and SharpView are suppressing noise and the image appearance is different compared to standard reconstruction. It is difficult to blind experienced readers to reconstruction techniques, due to different image texture. Because of the combination of image randomizing and focusing on assessing the image quality criteria on a five point 
scale, we were aiming at reducing the potential effect of recognition of the standard images familiar to the readers. In paper I and III physicists working with several CT scanners from all manufacturers were interpreting the images, so image recognition was a minor problem.

The European guidelines on quality criteria for computerized tomography applied in Paper II address normal structures in the thoracic and mediastinal CT rather than pathologic findings. This may be considered as a methodological limitation of this paper. It is, however, reasonable to assume that a higher ranking in VGC analysis in normal structures also will indicate a higher diagnostic performance for pathologic findings. Several studies published on diagnostic performance of post-processing filters and iterative algorithms used the same evaluation method as in this study ${ }^{32-35,51}$. This method is also described in a paper discussing different methods for evaluation of image quality ${ }^{14}$.

In paper II only full dose and one ultralow dose images were evaluated. Other dose levels were not assessed with respect to diagnostic performance following SharpView postprocessing. At the time of the study low dose CT thoracic examinations already were established at the radiological department at Oslo University Hospital, Ullevål, as standard procedure. Paper II demonstrated that SharpView post-processing improved the diagnostic performance of low dose examinations already established in the hospital. The exposure level of the low dose protocol used in paper II is comparable to dose levels used for assessing pulmonary nodules, as described in the literature ${ }^{59-66}$. The effect of SharpView on image quality for higher dose thoracic examinations is uncertain. To find exactly the dose reduction limit where the diagnostic performance is maintained using SharpView compared to the full dose level, scans at several dose levels should be obtained. This is hardly acceptable in clinical studies, because of increased radiation dose to patients. 


\subsection{The use of $\mathrm{HU}$ in diagnostics}

Each pixel reflects the linear radiation attenuation coefficient of the corresponding volume element (voxel) ${ }^{27}$. As new reconstruction techniques like iterative reconstruction are introduced, the measured HU may therefore differ substantially from those of FBP reconstruction. Also post-processing filters may influence on the measured $\mathrm{HU}$ of the images, due to differences in image texture.

HU measurements are widely used in diagnostics, especially in diagnostics of the lungs and abdomen. Using HU thresholds like "adrenal masses with density $\mathrm{HU}<10$ are supposed to be benign" is uncertain. In the specifications of the 64 slice CT scanners from all vendors, the $\mathrm{HU}$ of water is $0 \pm 4 \mathrm{HU}$, meaning that measurements in water could vary between $-4 \mathrm{HU}$ and $+4 \mathrm{HU}$ and still be under specification of the vendors ${ }^{68}$. This means that the vendors states that $\mathrm{HU}$ of water measured in a homogenous water phantom is $0 \pm 4 \mathrm{HU}$. In a patient such measurements normally deviate more due to beam hardening artefacts and partial volume artifacts. Besides, the differences in HU for specific tissue may differ substantially between CT scanners from different vendors according to differences in tube design, filtration and reconstruction among other factors. This interscanner variation was demonstrated in paper IV and also by Birnbaum et al. ${ }^{19}$. In Birnbaum's study, measured HU for simulated renal cysts in an anthropomorphic abdominal phantom varied between $1,5-10,2 \mathrm{HU}$ and the corresponding attenuation range was $39 \mathrm{HU}(-15,7,+23,9)^{19}$. These results underline the necessity of using relative HU measurements instead of absolute HU measurements for diagnostic purposes. 
Moreover, paper IV demonstrated that measured HU varied significantly between different energy levels on the same scanner. In particular, one manufacturer differed substantially from the others in this respect. The HU measurements performed on this scanner was lower than for the other vendors. As a consequence of our study the manufacturer developed a completely new reconstruction algorithm.

$\mathrm{HU}$ is highly energy dependent, which means that reducing the $\mathrm{kV}$ for an examination from 120 to 80 would affect the HU. Recently, publications on low $\mathrm{kV}$ in combination with ASIR have been published ${ }^{39}$. Still, radiologists use HU as absolute values for tissue characterization $^{22-30}$. Often, these thresholds are erroneously assumed to be both energyand scanner independent ${ }^{22}$. In example, Wilson et al. used a lung fraction limit of less than $-910 \mathrm{HU}$ to determine the presence of emphysema for $140 \mathrm{kVp}$ and $120 \mathrm{kVp}$. The measured HU of the same tissue will differ between these two energy levels, and would therefore not be comparable for diagnostic purposes. According to this, Wilson et al. should have used different $\mathrm{HU}$ limits for the $120 \mathrm{kVp}$ and the $140 \mathrm{kVp}$ level in characterizing emphysema.

Radiologists should use absolute CT-numbers with great care in diagnostics of their patients. It is recommended to use relative $\mathrm{HU}$ values in diagnostics of liver cysts and adrenals, not the absolute values, and also to compare the measured HU values to those of the normal tissue (cyst with the neighboring tissue). Still, the relative HU may vary slightly according to patient size due to beam hardening effects.

The Catphan phantoms used in paper IV are widely used by physicists for CT scanner performance tests. It contains sensitometry inserts in the range of $-1000 \mathrm{HU}$ to $990 \mathrm{HU}$. 
Still, it is not an anthropomorphic phantom, so the test inserts is not tissue equivalent. In this study the aim was to investigate the HU characteristics of the phantom and to measure potential inter-scanner variations in HU. With respect to this aim the phantom was suitable. 


\section{Conclusion}

The main goal of this work was to assess the impact of reconstruction- and post-processing filter on diagnostic performance, and evaluate the reliability of using absolute $\mathrm{HU}$ for diagnostic purposes.

The results from this thesis indicate that both ASIR and SharpView may be useful tools in improving the diagnostic performance and reducing CT dose. The benefit of an accurate diagnosis must be balanced against radiation risk. Optimization of CT examinations with respect to diagnostic performance and radiation dose is not only warranted but also a legal requirement in Norway.

It is possible to reduce the radiation doses from CT liver examinations up to $30 \%$ whilst maintaining or even improving the diagnostic performance in the detection of 2-7 $\mathrm{mm}$ simulated liver lesions by using SharpView post- processing. The results from the clinical study indicate that use of SharpView may improve the diagnostic performance for low dose CT examinations, and could be a helpful tool in optimizing image quality

The evaluation of ASIR indicate that a blending of 50\% ASIR and FBP improved image quality of detection of small liver lesions in the range 2-7 $\mathrm{mm}$ in an anthropomorphic liver phantom, and thus yield a potential for reducing radiation dose. Dose reduction of up to $50 \%$ for abdominal CT using ASIR may be possible, as the diagnostic performance is maintained or even improved compared to regular filtered back projection. 
Substantial interscanner variations in HU were demonstrated. Also substantial differences in measured HU between different spectral energies were seen. The HU of one, specific manufacturer differed substantially from the others. As new reconstruction techniques like iterative reconstruction are introduced, the measured HU may differ substantially compared to the HU for FBP reconstruction. Despite substantial inter-scanner variation absolute HU values are used for tissue and fluid characterization. The use of scanner independent and energy independent absolute HU should be used with caution. Instead relative HU should be used in tissue and fluid characterization, such as the difference in HU between different organs or different fluids. 


\section{Future perspectives}

There has been a substantially increase in the number of patients undergoing CT scanning, leading to an increase in population dose. There is no reason to believe that the frequency of CT examinations will be reduced in the future. Therefore, the manufacturers need to further improve the CT technology and develop new features such as post-processing filters and iterative reconstruction algorithms in order to provide and improve the necessary image quality without increasing the radiation doses. Improved versions of real iterative reconstruction instead of model based, statistical iterative reconstruction, may provide additional decreases in image noise, image artefacts and radiation dose.

The diagnostic performance and diagnostic image qualtiy should always be assured with respect to the ALARA principle before new technology is introduced in ordinary clinical practice. Research should focus on methods to achieve adequate diagnostic image quality at an optimum radiation dose in clinical practice. New features like iterative reconstruction techniques and post-processing filters should be fully evaluated in clinical practice to assess the benefit on diagnostic performance and potential of dose reduction. Also other dose reduction strategies, like more efficient detectors and further improved automatic tube current- and $\mathrm{kVp}$-modulation should be further developed and assessed clinically.

New reconstruction filters and post-processing filters, may introduce pitfalls with respect to alterations in the images. Such alterations may unintendedly affect the diagnostic performance and will not be discovered if not tested thoroughly. The images appear differently to the radiologists as new, noise suppressing post-processing reconstruction filters or iterative reconstruction algorithms are introduced. Some readers in this study 
commented that the filtered images looked unfamiliar or "strange". Further tests should be performed to fully evaluate the benefit to diagnostic performance and potential of dose reduction when using new iterative reconstruction algorithms and new post-processing filters clinically.

National and international guidelines for the justification and optimization of CT examinations are warranted. International studies on the stochastic and deterministic effects of radiation doses from radiological examinations are warranted. The use of the linear-nothreshold hypothesis which is used today is based on epidemiology from people that have received higher radiation doses, like victims from Chernobyl, Hiroshima and Nagasaki. CT scanners have been widely used for radiological purposes for more than 30 years. Therefore, it is possible to do retrospective analysis of possible stochastic effects for patients who have been examined during these period and estimate the risk of stochastic and deterministic effects.

Radiologists, physicists and radiographers should adopt consistent strategies for optimizing image quality and limiting and if possible, reducing radiation doses to the patients in the future. Multi-professional collaboration is necessary to fully assess different scan parameter settings, reconstruction filter combinations and at the same time optimization of the iodine contrast to achieve adequate CT examinations with the lowest possible radiation dose. In our hospital, multi-professional CT groups already are established. Such groups are necessary to achieve the ALARA principle in diagnostic radiology in the future, since the radiological equipment in general, and the CT scanners in special, are becoming more and more technically complex. 


\section{References}

1. Imhof H. Development of CT imaging. MedicaMundi 2006; 50:1.

http://www.healthcare.philips.com/pwc_hc/main/about/assets/Docs/medicamundi/m m_vol50_no1/09_Imhof.pdf (11.02.2011)

2. Beckmann EC. CT scanning the early days. Br J Radiol 2006; 79: 5-8

3. Kalender WA. X-ray computed tomography. Phys Med Biol 2006; 51: R29-R43

4. McCollough CH, Primak AN, Braun N, Kofler J, Lifeng Y. Strategies for reducing radation dose in CT. Radiol Clin N Am 2009; 47: 27-40

5. Almén A, Friberg EG, Widmark A, Olerud HM. Radiology in Norway anno 2008. Trends in examination frequency and collective effective dose to the population. StrålevernRapport 2010:12. Østerås: Norwegian Radiation Protection Authority, 2010. (In Norwegian)

6. Brenner DJ, Hall EJ. Computed tomography -An increasing source of radiation exposure. N Engl J Med 2007; 357:2277-2284

7. Gonzales AB, Darby S. Risk of cancer from diagnostic X-rays: estimates for the UK and 14 other countries, Lancet 2004; 363: 345-351

8. Børretzen I, Lysdahl KB, Olerud HM. Radiology in Norway - examination frequency per 2002, trends in time, geographical variation and population dose. StrålevernRapport 2006:6. Østerås: Norwegian Radiation Protection Authority, 2006. (In Norwegian)

9. Bongartz G, Golding SJ, Jurik AG, Leonardi M et al. European Guidelines for Multislice Computed Tomography. Funded by the European Commission. Contract number FIGM-CT2000-20078-CT-TIP March 2004. 
http://www.msct.eu/PDF_FILES/Part_two_final_document_quality_criteria_CT$\underline{\text { TIP.pdf }}$ (09.02.2011)

10. The 2007 Recommendations of the International Commission on Radiological Protection ICRP Publication 103 Approved by the Commission in March 2007

11. European Society of Radiology (ESR). White paper on radiation protection by the European Society of Radiology. Insights Imaging 2011. DOI 10.1007/s13244-0110108-1

12. Sodicksom A, Baeyens PF, Andriole KP, Prevedello LM et al. Recurrent CT, cumulative radiation exposure, and associated radiation-induced cancer risks from CT of adults. Radiology 2009; 251: 175-184

13. Berrington de Gonzales A, Mahesh M, Kim KP, Bhargavan M et al. Projected cancer risks from Computed Tomographic scans performed in the United States in 2007. Arch Intern Med 2009; 169: 2071-2077

14. Månsson LG. Methods for the evaluation of image quality: A review. Rad Protect Dosim 2000; 90: 89-99

15. Summers RM. Dose reduction in CT: The time is now. Acad Radiol 2010; 17 : 1201-1202

16. Sæther HK, Martinsen ACT, Korsmo L, Reisether T. Radiation protection of childrens' chest during high resolution CT of the lungs. Tidsskr Nor Legeforen, 2009; 129: 521-523 (in Norwegian)

17. Mayo JR, Aldrich J, Müller NL. Radiation exposure at chest CT: A statement of the Fleischner society. Radiology 2003; 228:15-21

18. Kalra MK, Maher MM, Toth TL, Blake MA et al. Strategies for CT radiation dose optimization. Radiology 2004; 230: 619-628 
19. Birnbaum BA, Hindman N, Lee J, Babb JS. "Multi-Detector Row CT Attenuation Measurements: Assessment of Intra- and Interscanner Variability with an Anthropomorphic Body CT Phantom,” Radiology 2007; 242: 109-119

20. Levi C, Gray JE, McCollough EC, Hattery RR. The Unreliabitility of CT numbers as absolute Values. AJR 1982; 139:443-447

21. Zerhouni EA, Boukadoum M, Siddiky MA, et al. A Standard Phantom for Quantitative CT Analysis of Pulmonary Nodules. Radiology 1983; 149:767-773

22. Hamrahian AH, Ioachimescu AG, Remer EM, Motta-Ramirez G et al. Clinical Utility of Noncontrast Computed Tomography Attenuation Value (Hounsfield Units) to Differentiate Adrenal Adenomas/Hyperplasias from Nonadenomas: Cleveland Clinic Experience. J Clin Endocrinol Metab 2005; 90: 871-877

23. Boland GWL, Lee MJ, Gazelle GS, Halpern EF et al. Characterization of Adrenal Masses Using Unenhanced CT: An analysis of the CT Literature. AJR 1998; 171: 201-204

24. Korobkin M. CT Characterization of Adrenal Masses: The Time Has Come. Radiology 2000; 217: 629-632

25. Yajima Y, Narui Y, Ishii M, Abe R et al. Computed Tomography in the Diagnosis of Fatty Liver: Total Lipid Content and Computed Tomography Number. Tohuko J exp Med 1982; 136: 337-342

26. Bosniak MA. The Current Radiological Approach to Renal Cysts. Radiology 1986; 158: $1-10$

27. Blay JY, Bonvalot S, Casali P, Choi H, Debiec-Richter M et al. Consensus meeting for the management of gastrointestinal stromal tumors. Report of the GIST Consensus Conference of 20-21 March 2004, under the auspices of ESMO. Annals of Oncology 2005; 16:566-578 
28. Nieman LK. Approach to the patient with an adrenal incidentaloma. J Clin Endocrinol Metab 2010; 95:4106-4113

29. Illias I, Sahdev A, Reznek RH, Grossmann AB, Pacak K. The optimal imaging of adrenal tumours: a comparison of different methods. Review. Endocrine-Related Cancer 2007; 14:587-599

30. Wilson DO, Leader JK, Fuhrman CR, Reilly JJ et al. Quantitative computed tomography analysis, airflow obstruction, and lung cancer in the Pittsburgh lung screening study. J Thorac Oncol 2011; 6; PMID: 21610523 (published ahead of print)

31. Skaane P, Huebener K-H. Computed tomography of cystic ovarian teratomas with gravity-dependent layering. Journal of Computer Assisted Tomography 1983; $7: 837-841$

32. Sing S, Kalra MK, Hsieh J, Licato PE et al. Abdominal CT: Comparison of adaptive statistical iterative and filtered back projection reconstruction techniques. Radiology 2010; 257: 373-383

33. Prakash P, Kalra MK, Kambadakone AK et al. Reducing abdominal CT radiation dose with adaptive statistical iterative reconstruction technique. Invest Radiol 2010; 45: $202-210$

34. Fleischmann D, Boas EF. Computed tomography -old ideas and new technology. Eur Radiol 2011; 21:510-517

35. Sagara Y, Hara AH, Silva AC, Paden RG et al. Abdominal CT: Comparison of lowdose CT with adaptive statistical iterative reconstruction and routine-dose CT with filtered back projection in 53 patients. AJR 2010: 195; 713-719

36. Hsieh J. Computed Tomography: Principles, Design, Artefacts, and recent advances. $2^{\text {nd }}$ ed. Bellingham, WA: SPIE press; 2009. Chapter 3: Image reconstruction, page 101-112 
37. Leander P, Söderberg M, Fält T, Gunnarson M et al. Post-processing image filtration enabling dose reduction in standard abdominal CT. Radiat Prot Dosimetry 2010; 139: 180-185

38. Kalra MK, Maher MM, Sahani DV, Blake MA et al. Low-dose CT of the abdomen: Evaluation of image improvement with use of noise reduction filters -pilot study. Radiology 2003; 228: 251-256

39. Marin D, Nelson RC, Schindera ST, Youngblood RS et al. Low-tube-voltage, Hightube-current multi-detector abdominal CT: Improved image quality and decrased radiation dose with adaptive statistical iterative reconstruction algorithm -initial clinical experience. Radiology 2010; 254: 145-153

40. Kröpil P, Lanzman RS, Walther C, Röhlen S et al. Dose reduction and image quality in MDCT of the upper abdomen: Potential of an adaptive post-processing filter. Fortschr Röntgenstr 2009; 181: 248-253 (in German)

41. Leipsic J, LaBounty TM, Heilbron B, Min JK et al. Adaptive statistical iterative reconstruction: Assesment of image nosie and image quality in coronary CT angiography. AJR 2010; 195:649-654

42. Silva AC, Lawder HJ, Hara A, Kujak J, Pavlicek W. innovations in CT dose reduction strategy: Application of the adaptive statistical iterative reconstruction algorithm. AJR 2010; 194:191-199

43. Leipsic J, Heilbron BG, Hague C. Iterative reconstruction for coronary CT angiography: finding its way. Int J Cardiovasc Imaging 2011; DOI 10.1007/s10554011-9832-3 (published ahead of print)

44. Leipsic J, LaBounty TM, Heilbron B, Min JK et al. Estimated radiation dose reduction using adaptive iterative statistical iterative reconstruction in Coronary $\mathrm{CT}$ angiography: The ERASIR study. AJR 2010; 195:655-660 
45. Hara AK, Paden RG, Silva AC, Kujak JL et al. Iterative reconstruction technique for reducing body radiation dose at CT: Feasibility study. AJR 2009; 193: 764-771

46. Carrino JA. Digital image quality: A clinical perspective. Quality assurance. Meeting the challenge in the digital medical enterprise. Chapter 3, SCAR, Great Falls Virgina 2002

47. Metz CE. ROC methodology in radiographic imaging. Invest Radiol 1986; 21: 72073

48. Metz CE. Some practical issues of experimental design and data analysis in radiological ROC studies. Invest Radiol 1989; 24: 234-245

49. Hanley JA, McNeil B J. The meaning and use of the area under a receiver operating characteristic (ROC) curve, Radiology 1982; 143:29-36

50. Olerud HM, Olsen J, Skretting A. An anthropomorphiv phantom for receiver operating characteristic studies in CT imaging of liver lesions. Br J Radiol 1999; $72: 35-43$

51. The Phantom Laboratory, Catphan 500 and 600 Manual, 7/26/10. http://www.phantomlab.com/pdf/catphan500-600manual.pdf (14.01.2011)

52. Nagel HD, Stumpp P, Kahn T, Gosh D. Performance of an automatic dose control system for CT: Specifications and basic phantom tests. Fortschr Röntgenstr 2011; 183: $60-67$

53. Widmark A, Friberg EG. Guidance No. 5b to "Regulations for radiation protection and use of radiation". Veileder om representative doser for røntgenunders $\varnothing$ kelser. Norwegian Radiation Protection Authorithy 2007. (in Norwegian). http://www.nrpa.no/dav/5184774fe5.pdf (06.02.2011) 
54. Method, Computer program product and apparatus for enhancing a computerized tomography image. Pt pending (No. WO 2005/091219 A1), ContextVision AB. 2005

55. CT dose reduction using SharpView CT, White paper version 1.2, 2005. Linköping: ContextVision AB; 2005. http://www.contextvision.se/ (5.12.2006)

56. Jurik A, Petersen J, Jessen KA, Bongartz G, et al. Clinical use of image quality criteria in computed tomography: A pilot study. Radiat Prot Dosimetry 2000; $90: 47-52$

57. Coakley FV, Gould R, Yeh BM, Arenson RL. CT radiation dose: What can you do right now in your practice? AJR 2011; 196: 619-625

58. Wessling J, Esseling R, Raupach R, Fockenberg S et al. The effect of dose reduction and feasibility of edge-presevring noise reduction on the detection of liver lesions using MSCT. Eur Radiol 2007; 17:1885-1891

59. Lubner MG, Pickhardt PJ, Tang J, Chen GH. Reduced image noise at low-dose multidetector $\mathrm{CT}$ of the abdomen with prior image constrained compressed sensing algorithm. Radiology 2011; doi:10.1148/radiol.11101380 (published ahead of print)

60. Wormanns D, Ludwig K, Beyer F, Heindel W, Diederich S. Detection of pulmonary nodules at multirow-detector $\mathrm{CT}$ : effectiveness of double reading to improve sensitivity at standard-dose and low-dose chest CT. Eur Radiol 2005; $15: 14-22$

61. Diederich S, Lenzen H, Windmann R, Puskas Z et al. Pulmonary nodules: Experimental and Clinical Studies at low-dose CT. Radiology 1999; 213:289-298 
62. Karabu.ut N, Törü M, Gelebek V, Gülsün M, Ariyürek O M. Comparison of lowdose and standard-dose helical CT in the evaluation of pulmonary nodules. Eur Radiol 2002; 12:2764-2769

63. Menezes RJ, Roberts HC, Paul NS, McGregor M et al.Lung cancer screening using low-dose computed tomography in at-risk individuals: The Toronto experience. Lung Cancer 2010; 67:177-183

64. Li X, Eshan S, DeLong D, Jones RP et al. Paediatric MDCT: Towards Assessing the Diagnostic Influence of Dose Reduction on the Detection of Small Lung Nodules. Acad Radiol 2009;16:872-880

65. Pedersen JH, Ashraf H, Dirksen A, Bach K et al. The Danish Randomized Lung Cancer CT screening Trial -Overall Design and Results of the Prevalence Round. J Thorac Oncol 2009; 4:608-614

66. Linning E, Daqing M. Volumetric Measurement Pulmonary Ground-Glass Opacity Nodules with Multi-detector CT: Effect of Various Tube Current on Measurement Accuracy -A Chest CT Phantom Study. Acad Radiol 2009;16:934-939

67. Brenner DJ. Radiation risk potentially associated with low dose CT screening of adult smokers for lung cancer. Radiology 2004; 232:440-445

68. International standard IEC 61223-3-5. Evaluation and routine testing in medical imaging departments- Part 3-5: Acceptance tests - Imaging performance of computed tomography X-ray equipment. First edition, aug. 2004 
9 Papers I-IV 



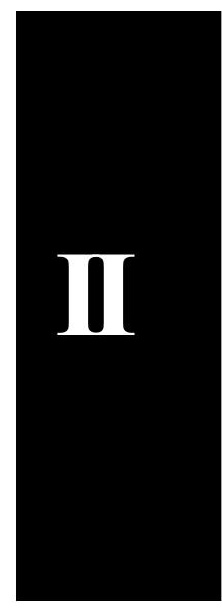





\title{
Improved image quality of low-dose thoracic CT examinations with a new postprocessing software*
}

\author{
Anne Catrine Traegde Martinsen, ${ }^{1,2,3 a}$ Hilde Kjernlie Saether, ${ }^{1,2,3}$ \\ Dag Rune Olsen, ${ }^{4,5}$ Per Aage Wolff, ${ }^{3}$ Per Skaane, ${ }^{2,3}$ \\ Interventional Centre, ${ }^{1}$ Oslo University Hospital, Oslo, Norway; Institute of Hospital \\ Medicine, ${ }^{2}$ University of Oslo, Norway; Department of Radiology, ${ }^{3}$ Oslo University \\ Hospital, Oslo, Norway; Institute of Cancer Research, ${ }^{4}$ Oslo University Hospital, Oslo, \\ Norway; Department of Physics, ${ }^{5}$ University of Oslo, Oslo, Norway. \\ anne.catrine.martinsen@ous-hf.no
}

Received 16 December, 2009; accepted 8 April, 2010

In 2008 a phantom study indicated that there is a potential for reducing the CT doses when using a new postprocessing filter. The purpose of this study was to test this new postprocessing filter clinically for low-dose chest CT examinations, to assess whether the diagnostic performance is the same or improved. A standardized clinical chest CT protocol was used on patients with colorectal cancer. Only $\mathrm{mA}$ settings changed between patients according to patient size. One standard and one low-dose chest protocol were performed for all patients. The low-dose images were postprocessed with a new software filter, which provides context-controlled restoration of digital images by using adaptive filters. Three radiologists assessed randomly all the images independently. A total of 24 scan series were evaluated with respect to image quality according to quality criteria from the European guidelines for chest CT using a five-point scale; 576 details were assessed. Overall mean score is the average score for all details rated for all three readers for all full-dose series, low-dose series and low-dose enhanced series, respectively. The statistical methods used for comparison were paired sampled t-test and intraclass correlation coefficient. The postprocessing filter improved the diagnostic performance compared to the unenhanced low-dose images. Mean score for full-dose, low-dose and low-dose enhanced series were 3.8, 3.0 and 3.3, respectively. For all patients the full-dose series gave higher scores than the low-dose series. Intraclass correlation coefficients were $0.2,0.1$ and 0.3 for the full-dose, low-dose and low-dose enhanced series, respectively. There is a potential for improving diagnostic performance of low-dose CT chest examinations using this new postprocessing filter.

PACS number: 87.57.C-, 87.57.Q-

Key words: CT, low-dose CT, chest CT, postprocessing filter

\section{INTRODUCTION}

Fourteen percent of total worldwide exposure to radiation is from diagnostic X-ray exposure. ${ }^{(1)}$ In European and US hospitals, the CT examinations account for more than $50 \%$ of the collective effective dose ${ }^{(2-4)}$ associated with medical exposure.

For doses of $100 \mathrm{mGy}$ and higher, there is a proven risk for radiation-related cancer induction, and there is no rational for assuming a low-dose threshold for cancer induction. ${ }^{(5)}$ In radiation

\footnotetext{
a Corresponding author: Anne Catrine Martinsen, Interventional Centre, ${ }^{1}$ Oslo University Hospital, N-0027 Oslo, Norway; phone: +47 230701 11; fax: +47 230701 10; email: anne.catrine.martinsen@ous-hf.no

* This study was reviewed and approved by the Regional Committees for Medical Research Ethics and the Norwegian Radiation Protection Authority.
} 
protection it is, therefore, a general assumption that the risk for stochastic effects increases linearly with dose, without any threshold.

A British study indicates that about $0.6 \%$ of the cumulative risk of cancer in the UK could be attributable to diagnostic X-ray, equivalent to 700 cases. ${ }^{(1)}$ In the study, the estimated number of radiation-induced cases of lung cancers per year based on 1998 UK population was 61 for both sexes combined. Mayo et al. ${ }^{(6)}$ concludes that the complex relationship between radiation exposure, image noise and diagnostic accuracy should be investigated further to establish the minimum radiation dose required to provide adequate diagnostic image quality. The main goal in optimizing CT examinations is to reduce the radiation and at the same time maintain or even improve diagnostic accuracy.

In order to improve diagnostic image quality with respect to noise supression, low-contrast detectability and spatial resolution without, at the same time, increasing the radiation doses from CT examinations, some manufacturers have developed stand-alone postprocessing tools that are compatible with all commercially available CT scanners. A phantom study of CT examinations of liver lesions published in 2008, indicated a potential for reducing the doses by $30 \%$ and, at the same time, maintaining the diagnostic image quality by using a postprocessing filter called SharpView CT. ${ }^{(7)}$ This filter is applied as a postprocessing step between the scanner and the picture archive (PACS) after the image reconstruction on the CT scanner is finished, working on the processed image data not the raw data, from the scanner. The filter is intended for enhancing edges and lines, 2D adaptive noise suppression and artefacts, in addition to spatial consistency.

The aim of our study was to evaluate the postprocessing filter SharpView CT clinically for low-dose thoracic CT examinations, and to assess if the diagnostic performance is affected using this new filter. It was not expected that the image quality for the low-dose thoracic CT examinations would be at the same diagnostic level as the full-dose images; rather we wanted to assess if the diagnostic performance at the low-dose level was affected using this new filter, since low-dose thoracic CT exams are used more and more worldwide.

\section{MATERIALS AND METHODS}

A total of 13 colorectal cancer patients with suspected or already known thoracic metastasis were scanned. For five of the patients, the scan series did not cover all the chest area of interest in this study and they were consequently excluded from the study. All patients underwent standard CT scanning of liver and chest, as part of the national cancer follow-up regime. Eight patients were included in the data analysis; four male and four female, ranging in age from 50 to 75 years; mean age was 67.6 years.

The oncologists responsible for the treatment of each patient were informed about the study. All patients were included in the study upon informed consent. Each patient underwent one full-dose thoracic CT exam and one low-dose thoracic CT exam.

All CT protocols in the hospital had been optimized beforehand in order to minimize the dose levels while maintaining adequate diagnostic performance.

In this study, all scans were performed using a 64-slice CT scanner (Philips Brilliance 64, Best, The Netherlands). The scan parameters for the full-dose thoracic CT protocol were $120 \mathrm{kV}$, $0.7 \mathrm{~s} /$ rotation, pitch $0.9,64$ by $0.625 \mathrm{~mm}$ collimation and $200 \mathrm{mAs}$. In the hospital use of the automatic current selection (ACS) and dose modulation in Z-direction (Z-DOM) are standard. Mean pitch corrected values of weighted CT dose index $\left(\mathrm{CTDI}_{\mathrm{vol}}\right)$ was $8 \mathrm{mGy}$ for the patients included in the study. In comparison, the national reference level for chest CT in Norway is $\mathrm{CTDI}_{\mathrm{vol}}=20 \mathrm{mGy}^{(8)}$

The low-dose protocol applied in the study is based on the following parameters: $120 \mathrm{kV}$, $0.7 \mathrm{~s}$ /rotation, pitch 0.9 , collimation 64 by $0.625 \mathrm{~mm}$ and $30 \mathrm{mAs}$. ACS and Z-DOM were not used for the low-dose protocol. CTDI ${ }_{\text {vol }}$ for the low-dose scan was $1.8 \mathrm{mGy}$. The exposure 
level of the low-dose protocol used in the study is comparable to dose levels used for assessing pulmonary nodules, as described in the literature. ${ }^{(5,9-16)}$

The reconstructed images were $2 \mathrm{~mm}$ thick. Two physicists and a radiographer, who were not involved in the image evaluation process, performed all imaging and the postprocessing image reconstruction.

One full-dose and one low-dose thoracic CT scans were performed for all patients. The low-dose images were postprocessed using an adaptive filter named SharpView CT (SharpView AB, Linköping, Sweden). Figure 1 shows a full-dose chest image without SharpView postprocessing and a low-dose image with SharpView post-processing. Figure 2 shows two low-dose CT images with standard and with SharpView postprocessing. The image contrast is different for the full-dose and the low-dose images due to different radiation doses. During image assessing, the window width and window level were changed by the observers as the observers normally do in a clinical situation.

Intravenous iodine contrast was given for the full-dose scans for all patients. The image quality criteria evaluated in this study are not influenced by the use of iodine contrast enhancement.

Three observers, all cross-sectional radiologists, assessed the images independently. The observers were given a set of 24 scan series in total obtained at two different dose levels, $200 \mathrm{mAs}$ and $30 \mathrm{mAs}$. Both standard and SharpView postprocessing were evaluated at low dose. Only the standard postprocessing was evaluated for full-dose images.

Twenty-four scan series and eight image quality criteria were evaluated by the three observers; in total 576 details were assessed. All series were assessed with respect to eight CT image quality criteria from the European guidelines on quality criteria for CT on a five-point scale. ${ }^{(17)}$ The criteria were chosen from the list of general chest and HRCT chest criteria. Criteria for the mediastinal region, lung parenchyma and the lung tissue were chosen to assess the new postprocessing filter. Table 1 lists the criteria assessed in the study. CTDI recommended in the guidelines are 35 and $30 \mathrm{mGy}$ for the HRCT and the routine CT exam, respectively. Also the pitch corrected values of weighted CT dose index $\left(\mathrm{CTDI}_{\mathrm{vol}}\right)$ would be 35 and $30 \mathrm{mGy}$ respectively, which is higher than both the national reference value and the doses used in this study. In total, 505 out of 576 quality criteria details were possible to rate; the remaining were not rated due to too-short scan regions in some examinations. For some patients, certain details could consequently not be rated for all dose levels. The 505 details were included to form the basis for the statistical analyses in this study.

TABLE 1 . Image quality criteria.

The image quality criteria from European guidelines for chest CT used in this study. The observers gave a score on a scale from 1 to 5 , where score $1=$ not visible; score $2=$ poor/hardly visible; score $3=$ visible; score $4=$ clearly visible; score $5=$ visually sharp.

1: Reproduction of pulmonary fissures

2: Reproduction of small pulmonary vessels within $1 \mathrm{~cm}$ off the pleura

3: Reproduction of bronchial walls within $3 \mathrm{~cm}$ from the chest wall

4: Reproduction of major mediastinal vessels

5: Reproduction of endotracheal and endobronchial margins

6: Reproduction of trachea and central bronchial wall

7: Reproduction of lateral pleural margins

8: Reproduction of pleuromediastinal margin

Before the start of image assessment, all image quality criteria and the score scheme were explained in detail. Also the use of the quality criteria was demonstrated using sample images.

The observers were blinded to technical factors and imaging mode. To prevent learning bias, all images were randomly displayed on a review workstation. The reading process was performed in three sessions. Each reading session consisted of one scan series for each patient, randomized with respect to dose levels, such that a reading session consisted of full-dose series 

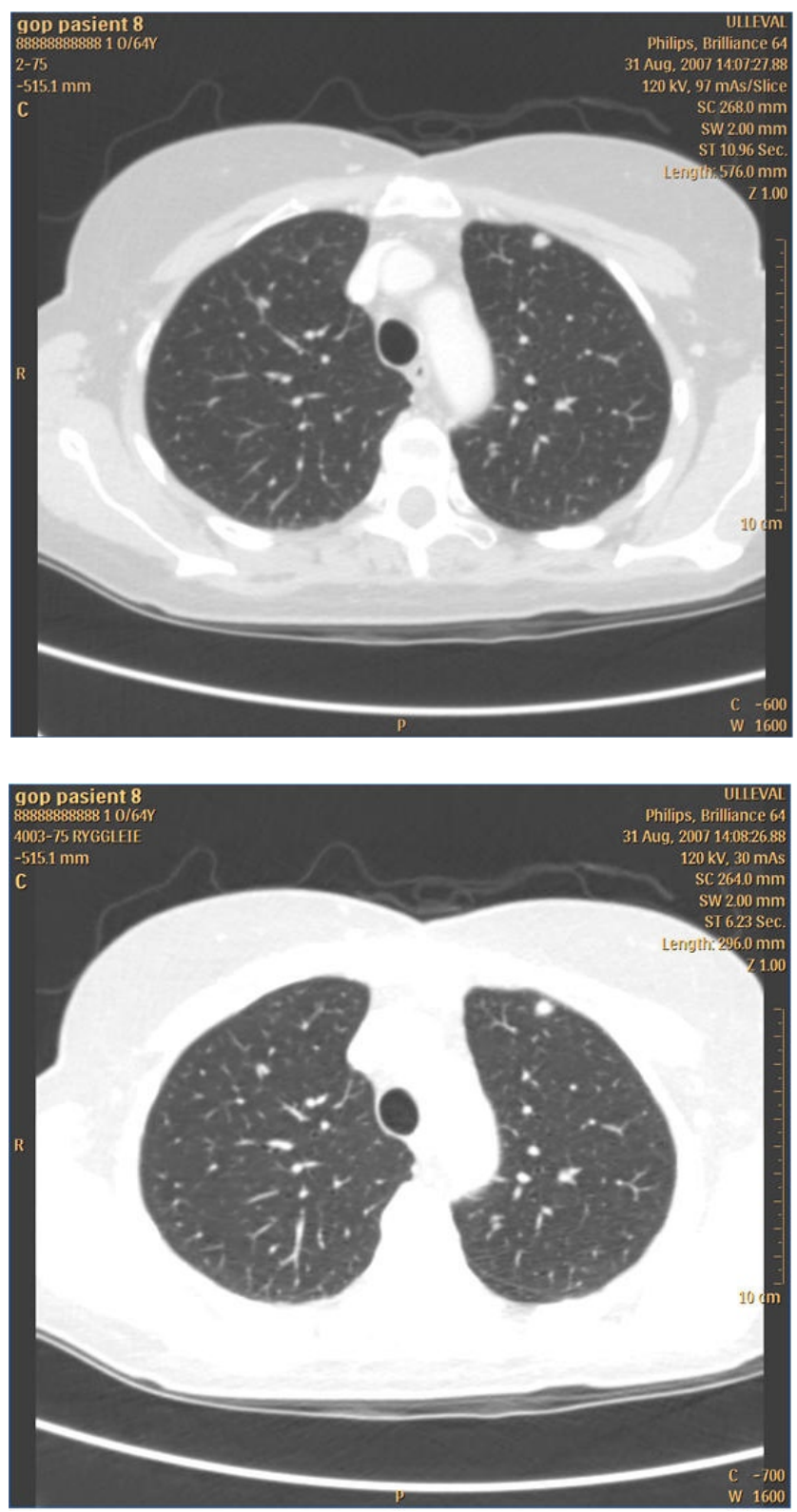

FIG. 1. A full-dose chest CT image (a) in which only standard reconstruction was used; a low dose chest CT image (b) for the same patient in which SharpView postprocessing filter was used.

Journal of Applied Clinical Medical Physics, Vol. 11, No. 3, Summer 2010 

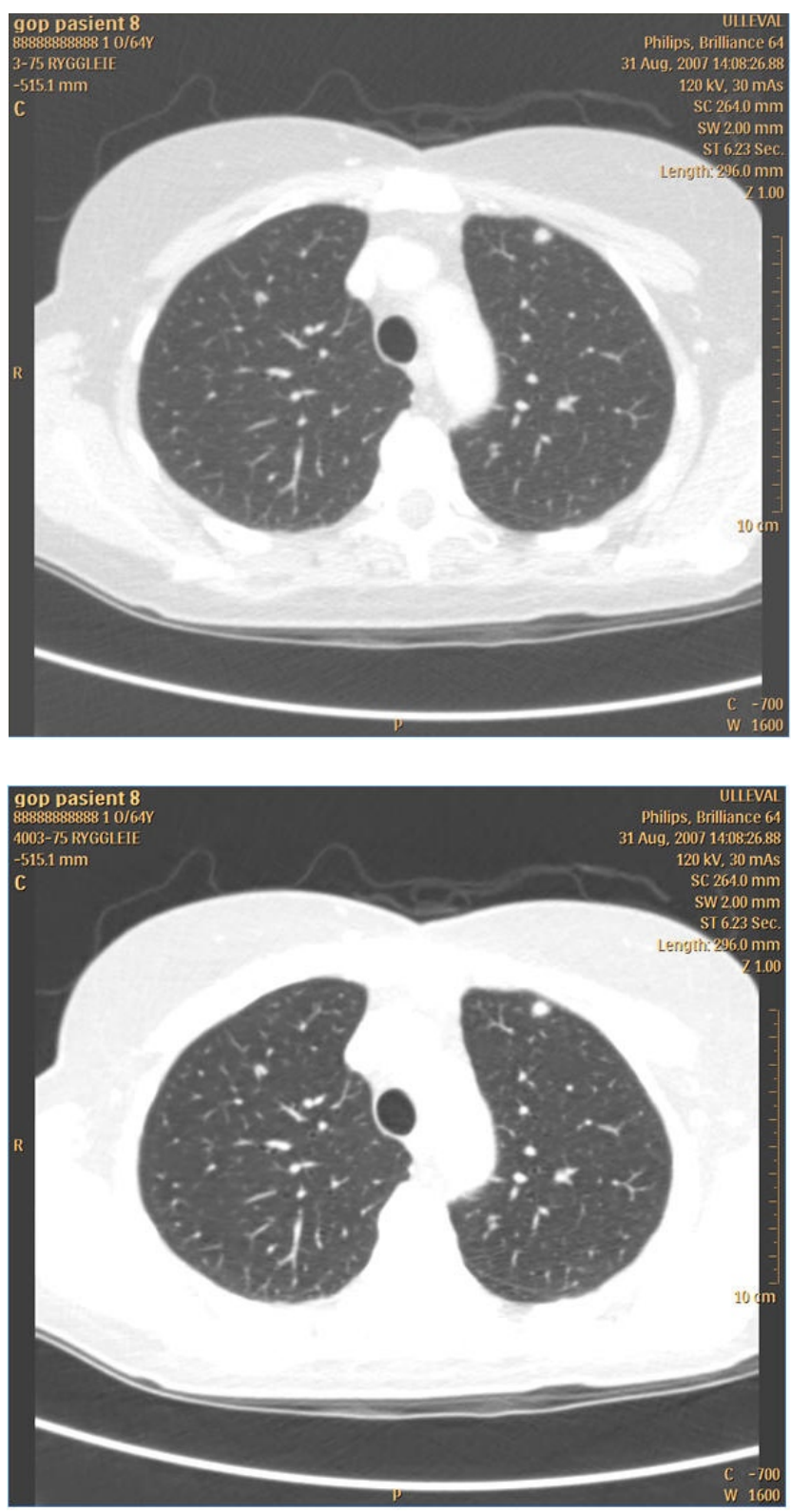

FIG. 2. Two low-dose chest CT images, at the same dose level, at the same position for the same patient: (a) standard reconstruction was used; (b) image SharpView postprocessing filter was used.

Journal of Applied Clinical Medical Physics, Vol. 11, No. 3, Summer 2010 
for some patients, low-dose series for some patients and low-dose SharpView enhanced series for some patients. Image reading included three reading sessions over 16 weeks for each reader, to avoid recognition of the pathology appearing in the images.

None of the observers were involved in the image acquisition process. All images were displayed on the same review workstation. No time constraints were given.

The SharpView CT postprocessing filter identifies image features at different abstraction levels using a hierarchical approach. ${ }^{(18)}$ The filter combines $2 \mathrm{D}$ adaptive noise suppression, edge enhancement and spatial resolution.

Different acquisition settings, such as dose and reconstruction algorithm, greatly affect the image data characteristics and might, therefore, require particular filter parameters. The parameters may also be adjusted to account for anatomical variations. The enhancement is performed in different intensity value ranges, corresponding to tissue type-specific Hounsfield Units (HU). ${ }^{(19)}$ The parameters can thus be set differently for different intensity ranges (tissues), making simultaneous filtering of soft and lung tissue possible in chest examination. The SharpView CT postprocessing filter was adjusted with respect to CT scanner, anatomical region of interest and dose levels to fit the thoracic $\mathrm{CT}$ images as perfectly as possible. Three different versions of postprocessing filter were tested before the study started, to ensure that the most optimal filter was used in the study.

The SharpView CT vendor had no role in study design, data collection, data analysis, data interpretation or in writing this paper, except the sentences describing the functionality of the Sharpview algorithm.

A paired sampled t-test with a $95 \%$ level of confidence was used to compare diagnostic performance of images with and without postprocessing image enhancement. Inter-observer differences were assessed by using intraclass correlation coefficient (ICC) test with $95 \%$ confidence interval. ICC ranges from 1 to $0: \mathrm{ICC}=1$ corresponds to complete agreement between the observers, and ICC $=0$ corresponds to no agreement at all between the observers.

The overall mean scores is the mean score for all details rated for all readers for all full-dose series, low-dose series and low-dose enhanced series, respectively.

\section{RESULTS}

The overall mean scores for all image quality details for all full-dose series, all low-dose series and all low-dose enhanced series for all three readers were 3.8, 3.0 and 3.3, respectively. The overall image quality scores for all examinations and observers was significantly higher for the full-dose series compared to both the low-dose series and enhanced low-dose series $(p<0.05)$ (Fig. 3). The overall mean scores for the SharpView enhanced low-dose series was higher than the overall mean scores for the low-dose series (3.3 versus 3.0, respectively, $p<0.05$ ).

The three observers consistently reported overall image quality of the full-dose series superior to that of both the low-dose series and the low-dose series with SharpView image quality enhancement (Fig. 4). The scores for the full-dose, low-dose and the low-dose SharpView postprocessed series were 3.6, 3.1 and 3.3 for reader one, 4.1, 2.4, and 3.2 for reader two, and $3.7,3.4$ and 3.5 for reader three. The difference in image quality score between reader 1 and 2 for the full-dose and the low-dose SharpView postprocessed series was not significant. For observer 3, the image quality scores were significantly higher for the full dose. For all observers, the SharpView postprocessed series gave a significantly higher image quality score than the low-dose standard (Fig. 4).

For each individual patient (except patient 2 and 3), the full-dose series had the highest mean scores. A paired t-test showed that the difference between full-dose and SharpView enhanced low-dose series is not statistically significant (Fig. 5) for five of the patients. The difference in score between full-dose and low-dose series is significant for all patients, except one $(p<0.05)$. 


\section{Mean score for all radiologists and all patients}

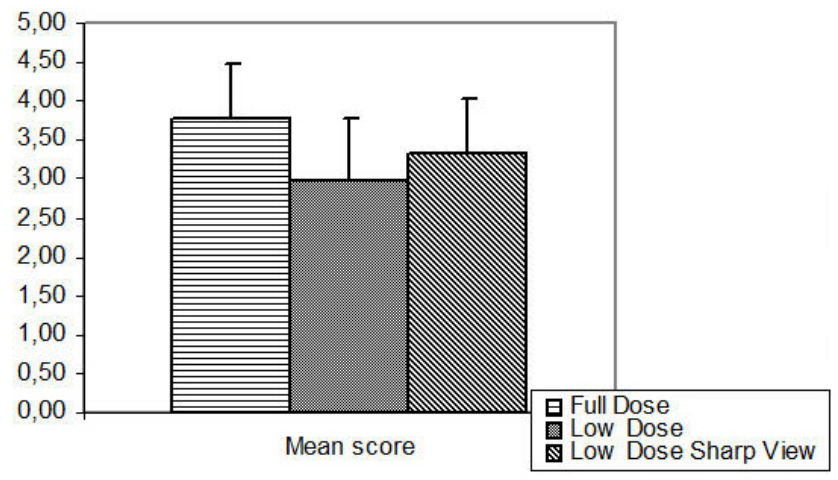

FIG. 3. Overall mean score (the average score for all details rated for all full-dose series, low-dose series and low-dose enhanced series) for all three readers. The full-dose series gave a significantly higher score than both the low-dose series and the SharpView enhanced low-dose series. The SharpView enhanced low-dose series gave a significantly higher score than the low-dose series.

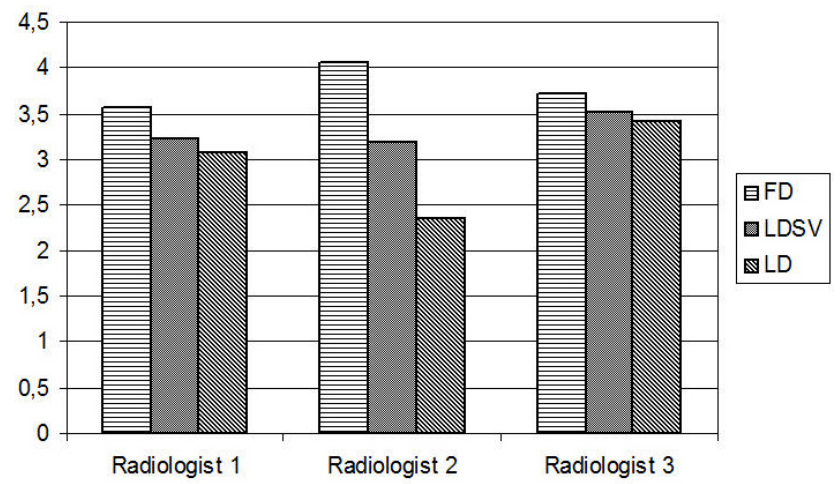

FIG. 4. Variation in overall score between radiologists. For all radiologists the total score is highest for the full-dose (FD) images and lowest for the low-dose (LD) images. SV is the SharpView postprocessed images; FD is the mean score for each radiologist of the full-dose series; LD is the mean score for each radiologist of the low-dose series.

The SharpView enhanced low-dose series gave a higher score than the low-dose series for all patients.

The full-dose series scored significantly higher than the other series for each image quality criteria. The scores for each image quality criteria ranged between 3.3 and 4.1 for the fulldose series, between 2.8 and 3.3 for the low-dose series, and ranged between 3.1 and 3.5 for the low-dose SharpView postprocessed series. SharpView postprocessed low-dose series had a higher score than the low-dose series for all image quality criteria. The difference in scores were significant for criterion 2: "Reproduction of small pulmonary vessels within $1 \mathrm{~cm}$ off the pleura" (score 3.2 versus 2.9 for the low-dose SharpView enhanced and the low-dose 


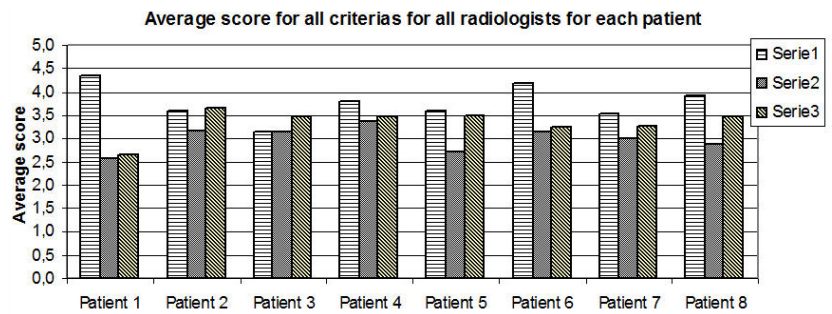

FIG. 5. The mean score for all criteria for all radiologists for each patient. For all patients, the full-dose series gave a higher score than the other two series (except for patient 2 and 3), and the low-dose SharpView enhanced series gave higher score than the low-dose series.

series, respectively, $p<0.05$ ), criterion 4: "Reproduction of major mediastinal vessels" (score 3.7 versus 3.2 for the low-dose SharpView enhanced and the low-dose series, respectively, $p<0.05$ ), criterion 5: "Reproduction of endotracheal and endobronchial margins" (score 3.5 versus 3.0 for the low-dose Sharpview enhanced and the low dose series respectively, $\mathrm{p}<0.05$ ) and criterion 8 "Reproduction of pleuromediastinal margin" (score 3.4 versus 2.9 for the low dose SharpView enhanced and the low-dose series, respectively, $p<0.05$ ). (See Table 1 and Fig. 6.) The difference was not significant for the criteria: "Reproduction of pulmonary fissures", "Reproduction of bronchial walls within $3 \mathrm{~cm}$ from the chest wall", "Reproduction of trachea and central bronchial wall" and "Reproduction of lateral pleural margins".

The mean score for all patients and all image quality criteria for each radiologist were above 3 for both the full-dose and low-dose SharpView enhanced series. The mean scores for all patients and all image quality criteria for the full-dose series were 3.6, 4.1 and 3.1 for reader 1, 2 and 3, respectively. The mean score for low-dose series were 3.1, 2.4 and 3.4 and the mean scores for low-dose SharpView enhanced series were 3.3, 3.2 and 3.5 for reader 1, 2 and 3, respectively (Fig. 7). For the low-dose series, the mean scores ranged between 2.4 and 3.4. The full-dose series had the highest scores for all observers. Observer 3 had higher scores than the other observers for both the low-dose series and the low-dose SharpView postprocessed series. (Observer 3 is a specialist in chest CT.) SharpView postprocessing resulted in the best agreement between the observers. Intraclass correlation coefficients (ICC) were 0.2 for full-dose images, 0.1 for low-dose images and 0.3 for SharpView low-dose images. 


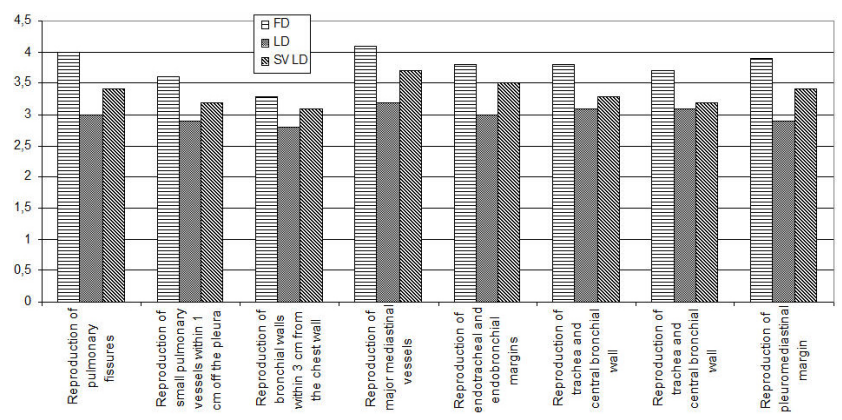

FIG. 6. The mean score for all radiologists and patients for each quality criteria. For all criteria, the full-dose series gave the significantly highest score (except for patient 2 and 3), and the low-dose SharpView enhanced series gave higher score than the low-dose series. The difference between the SharpView enhanced series and the low-dose series is not significant.

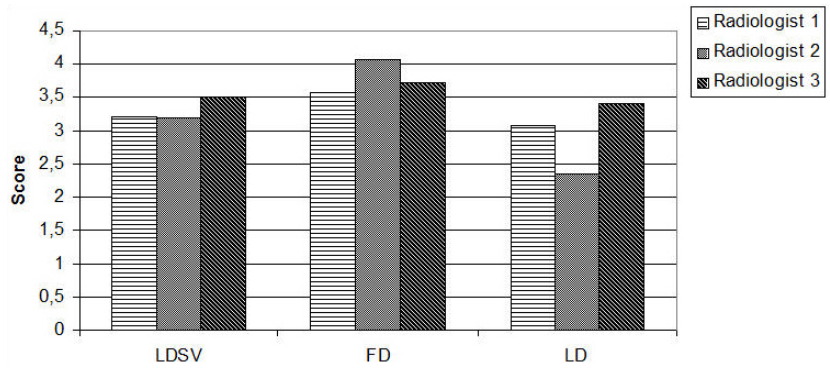

FIG. 7. The interobserver differences for the different dose levels. LDSV is the mean score for each radiologist of the low-dose SharpView enhanced series; FD is the mean score for each radiologist of the full-dose series; LD is the mean score for each radiologist of the low-dose series.

\section{DISCUSSION}

A previous study on the possible diagnostic performance of SharpView using a liver phantom demonstrated that the image quality was the same or even improved at $30 \%$ reduction in dose when using SharpView. ${ }^{(7)}$ In this study, SharpView was tested clinically at low-dose levels and compared to full-dose images without SharpView postprocessing.

The low-dose series always had a lower score than the SharpView enhanced series, indicating that the use of postprocessing filters like SharpView may improve the diagnostic image quality significantly for thoracic CT exams.

The difference in average score between low-dose and full-dose series for all observers, patients and image quality criteria is 0.8 (score 3.8 for full-dose and 3.0 for low-dose) (Fig. 3). This difference in diagnostic performance has to be evaluated against the fact that full-dose series gives six times the dose of the low-dose series. The modest increase in image quality using the full-dose protocol may not defend the larger dose as compared to the low-dose protocol combined with SharpView image enhancement.

The low-dose images generally had a lower score than the full-dose images, indicating that the low-dose images required image enhancement to provide the same diagnostic performance 
as the full-dose images. The SharpView enhanced low-dose series were rated significantly higher than the low-dose standard series. This may indicate that postprocessing filters like Sharpview could be a helpful tool in optimizing image quality.

All observers reported the highest mean score for the full-dose series and lowest mean score for the low-dose series. The mean score amongst the observers was most similar for the lowdose SharpView enhanced series, and also the mean score was higher for SharpView enhanced series compared to the low-dose series.

The interobserver differences were smaller for the low-dose SharpView enhanced series than for both the full-dose and the low-dose series. Reader 2 had the highest score for the full-dose series and lowest score for the low-dose series compared to the other readers, but scored the low-dose SharpView enhanced series equal to that of the others. This indicates a higher agreement between observers for the low-dose SharpView enhanced protocol.

In a pilot multicenter study, the European guidelines on image quality criteria for CT were tested with regard to their utility in clinical practice for thoracic $\mathrm{CT}$. The results from this study showed that the diagnostic criteria could be used to optimize CT procedures with respect to image quality and dose. ${ }^{(20)}$ Still, these criteria differ from normal diagnostic findings for a radiologist, and might seem unfamiliar to evaluate, and this might be the reason for the results of the ICC test where the score for the full-dose series used in clinically practice in the hospital today were as low as 0.2. For some series, the dose levels recommended in these European guidelines are above the national reference doses for chest CT, and higher than the doses used in this study. Motion artifacts due to cardiac pulsation and breathing could also be factors influencing the observers' scores and the interobserver differences.

To utilize the full capacity of postprocessing strategies, identifying the maximum dose reduction without compromising the equal image quality would be required. Assessing image at several dose levels can identify this threshold dose. Multiple CT examinations would then be required, but would obviously not be acceptable from an ethical or radiation protection point of view. In the literature, low-dose thoracic CT exams are performed as low as $30 \mathrm{mAs}$; this is the dose level used in our hospital. Especially for the mediastinal windows, the image quality may then in some cases be inadequate. To improve the image quality at this low dose level, SharpView postprocessing software may be one tool.

The image quality criteria chosen in this study should not be influenced by the fact that iodine contrast enhancement was utilized for the full-dose series. The image appearance differed, however, and other pathology might be visualized better, and thus the diagnostic performance might have been improved. The low-dose series and the low-dose SharpView enhanced series are processed with the same raw data, and are thus identical except for the postprocessing.

For the evaluation of score for each individual patient and for each individual image quality criteria, the sample sizes were smaller than for the other results in the study. Therefore, these significance tests should be interpreted with care. A larger clinical trial should be performed.

One of the radiologists is a specialist in chest CT, while the other two are general radiologist. The results varied less between different dose levels and postprocessing for the thoracic radiologist compared to the others. This might indicate that the learning process of this study should have been expanded to reduce interobserver differences.

\section{CONCLUSIONS}

In conclusion, using SharpView CT optimized with respect to anatomical region of interest and dose levels, gives a potential for improving diagnostic performance of low-dose CT thoracic examinations. 


\section{REFERENCES}

1. Berrington de Gonzales A, Darby S. Risk of cancer from diagnostic X-rays: estimates for the UK and 14 other countries. Lancet. 2004;363(9406):345-51.

2. Jessen KA. Going digital can help lower radiation dose. Diagnostic Imaging. 2004. Available from: http://www. diagnosticimaging.com/pacsweb/showArticle.jhtml?articleID $=51202507$

3. Mettler FA, Wiest PW, Locken J, Kelsey C. CT scanning: patterns of use and dose. J Radiol Prot. 2000;20(4):353-59.

4. Børretzen I, Lysdahl KB, Olerud HM. [Radiology in Norway - examination frequency per 2002, trends in time, geographical variation and population dose.] Østerås, Norway: Norwegian Radiation Protection Authority; 2006 .

5. The 2007 Recommendations of the International Commission on Radiological Protection. ICRP Publication 103. Ann ICRP. 2007;37(2):1-332.

6. Mayo JR, Aldrich J, Müller NL. Radiation exposure at chest CT: a statement of the Fleischner Society. Radiology. 2003;228(1):15-21.

7. Martinsen ACT, Sæther HK, Olsen DR, Skaane P, Olerud HM. Reduction in dose from CT examinations of liver lesions with a new postprocessing filter: a ROC phantom study. Acta Radiol. 2008:49(3):303-09.

8. Friberg EG, Widmark A, Olerud HM, Tynes T, Saxebøl G. [Guidance for use of medical X-ray and MR equipment subjected to approval. Guidance to "Regulations for radiation protection and use of radiation". Guidance No. 5.$]$ In Norwegian. Østerås: Norwegian Radiation Protection Authority, 2005. Available from: http://www.nrpa.no/ dav/bac3c61794.pdf

9. Wormanns D, Ludwig K, Beyer F, Heindel W, Diederich S. Detection of pulmonary nodules at multirow-detector CT: effectiveness of double reading to improve sensitivity at standard-dose and low-dose chest CT. Eur Radiol. 2005;15(1):14-22.

10. Diederich S, Lenzen H, Windmann R, et al. Pulmonary nodules: experimental and clinical studies at low-dose CT. Radiology. 1999;213(1):289-98.

11. Karabulut N, Törü M, Gelebek V, Gülsün M, Ariyürek O M. Comparison of low-dose and standard-dose helical $\mathrm{CT}$ in the evaluation of pulmonary nodules. Eur Radiol. 2002;12(11):2764-69.

12. Menezes RJ, Roberts HC, Paul NS, et al. Lung cancer screening using low-dose computed tomography in at-risk individuals: the Toronto experience. Lung Cancer. 2010;67(2):177-83.

13. Li X, Eshan S, DeLong D, et al. Paediatric MDCT: towards assessing the diagnostic influence of dose reduction on the detection of small lung nodules. Acta Radiol. 2009;16(7):872-80.

14. Pedersen JH, Ashraf H, Dirksen A, et al. The Danish randomized lung cancer CT screening trial - overall design and results of the prevalence round. J Thorac Oncol. 2009;4(5):608-14.

15. Linning E, Daqing M. Volumetric measurement pulmonary ground-glass opacity nodules with multi-detector CT: effect of various tube current on measurement accuracy - a chest CT phantom study. Acta Radiol. 2009;16(8):934-39.

16. Brenner DJ. Radiation risk potentially associated with low-dose CT screening of adult smokers for lung cancer. Radiology. 2004;231(2):440-45.

17. European Guidelines on Quality Criteria for Computed Tomography, EUR 16262. Available from http://www. drs.dk/guidelines/ct/quality/index.htm

18. Granlund GH. In search of a general picture processing operator. Computer Graphics and Image Processing. $1978 ; 8: 155-73$

19. Leander P, Söderberg M, Fält T, Gunnarasson M, Albertsson I. Post-processing image filtration enabling dose reduction in standard abdominal CT. Radiat Prot Dosim. 2010. Pub. online March 5, 2010.

20. Jurik A, Petersen J, Jessen KA, et al. Clinical use of image quality criteria in computed tomography: a pilot study. Radiat Prot Dosim. 2000;90(1-2):47-52. 



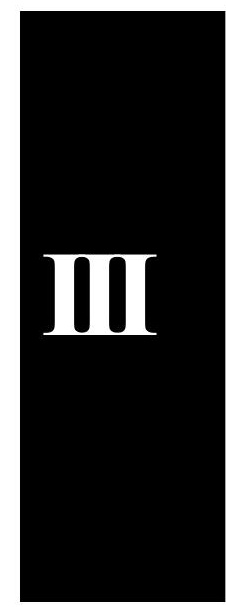





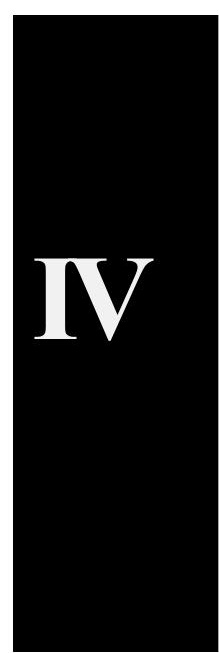


